\title{
Differential Modulation With Threshold-Based Decision Combining for Cooperative Communications
}

\author{
Thanongsak Himsoon, Member, IEEE, W. Pam Siriwongpairat, Member, IEEE, Weifeng Su, Member, IEEE, and \\ K. J. Ray Liu, Fellow, IEEE
}

\begin{abstract}
Differential modulation is widely known as a practical alternative that provides a good tradeoff between receiver complexity and performance. However, the available differential schemes for wireless relay networks require perfect synchronization and/or provide limited transmission rates. This paper proposes a threshold-based differential decode-and-forward cooperative scheme that not only alleviates the problems of synchronization and rate limitation, but also efficiently exploits the cooperative relay channels via the use of a predetermined decision threshold. In the proposed scheme, the source information is forwarded by the relay only if it is correctly decoded. The properly designed threshold enables the destination to decide whether the received signal from the relay contains information such that the received signals from the source and the relay can be efficiently combined and jointly decoded. Bit error rate (BER) performance analysis of the proposed scheme is analyzed in the case of differential M-ary PSK signals. The analysis focuses on the case when the relay is able to judge whether each decoded symbol is corrected or not. The obtained BER performance in this case serves as a performance benchmark, and the proposed differential decode-and-forward scheme can achieve it in an ideal situation that the error propagation at the relay can be negligible. A tight BER approximation is first derived, and then BER upper bound and lower bound are determined. Based on the tight BER approximation, joint optimum decision threshold and power allocation are numerically evaluated. Both analytical and simulation results reveal that the decision threshold and the power allocation depend on qualities of the communication channels. Interestingly, when the link quality between relay and destination is very good, the effect of the threshold dominates the effect of the power allocation at high signal-to-noise ratio. Extensive simulation results are provided to validate the merit of the proposed scheme and confirm the theoretical analysis.
\end{abstract}

Index Terms-Bit error rate (BER), cooperative diversity, decode-and-forward protocol, differential modulation, performance analysis, virtual multiple-input multiple-output (MIMO), wireless networks.

Manuscript received August 16, 2005; revised November 15, 2006. This work was supported in part by the U.S. Army Research Laboratory under Cooperative Agreement DAAD 190120011. The associate editor coordinating the review of this manuscript and approving it for publication was Dr. Ananthram Swami.

T. Himsoon and W. P. Siriwongpairat are with the Department of Electrical and Computer Engineering and the Institute for Systems Research, University of Maryland, College Park, MD 20742 USA, and also with Meteor Communications Corporation, Kent, WA 98032 USA (e-mail: khimsoon@meteorcomm. com; psiriwongpairat@meteorcomm.com).

W. Su is with the Department of Electrical Engineering, State University of New York (SUNY) at Buffalo, Buffalo, NY 14260 USA (e-mail: weifeng@eng. buffalo.edu).

K. J. R. Liu is with the Department of Electrical and Computer Engineering and the Institute for Systems Research, University of Maryland, College Park, MD 20742 USA (e-mail: kjrliu@eng.umd.edu).

Color versions of one or more of the figures in this paper are available online at http://ieeexplore.ieee.org.

Digital Object Identifier 10.1109/TSP.2007.894268

\section{INTRODUCTION}

D IFFERENTIAL detection, together with diversity combining, is widely known as an attractive alternative to coherent detection that provides a good tradeoff between receiver complexity and performance in conventional communication systems. The technique is also useful for sufficiently severe environment where phase and amplitude tracking for coherent demodulation is difficult if not impossible. In differential phase-shift keying (DPSK) systems [1], the transmitter differentially encodes input information in adjacent phase difference of the two transmitted symbols before transmitting the encoded symbol over fading channel. While perfect channel state information is not required, an efficient differential decoding at the receiver relies on constant fading from one time sample to the next. Such a slow fading assumption appears to be realistic in many practical applications, and it is crucial for performance analysis.

No need for channel estimation has attracted many researchers to deploy differential detection in multiple-input multiple-output (MIMO) systems [2], [3]. The differential schemes in [2] and [3] omit the burden of multichannel estimation, while provide significant performance improvement without compromising bandwidth efficiency over that of conventional single antenna system. However, the deployment of multiantenna terminals may be difficult in some applications because the mobile terminals are practically small. Recently, an idea of resource sharing among users in wireless networks has been introduced as cooperative communication paradigm [4], [5]. By taking advantage of the broadcasting nature of the wireless networks, the cooperative communications is able to explore inherent spatial diversity through relay channels by utilizing different kinds of cooperation protocols.

In [4] and [6], two possible cooperation protocols were introduced according to relay processing: amplify-and-forward (AF) and decode-and-forward (DF) protocols. In the case of the AF protocol, while one user acts as a source node and transmits its information, other users in the networks can act as relay nodes. The relay nodes amplify the signal that they receive from the source and forward the amplified signal to the destination. For the DF protocol, on the other hand, the relays decode the received signal and then forward the decoded information symbol to the destination. Also, in [4] and [6], the outage probability of the two cooperation schemes were analyzed. The proposed works in [7] and [8] show an idea of user cooperation for a two-user CDMA systems. In these schemes, both users 
simultaneously transmit information by using an orthogonal code such that the effect of multiple access interference can be reduced. Another cooperation technique called coded cooperation was proposed in [9], in which the existing channel codes are incorporated into the cooperation. The coded cooperation achieves higher diversity by transmitting two portions of the coded symbol separately. Specifically, the first encoded portion is transmitted by the source itself, while the other portion is delivered by its partners or relay nodes. Recently, rigorous analysis on exact symbol error rate (SER) and optimum power allocation for the DF protocol have been proposed in [10] for two-user cooperation systems. The exact SER formulation and optimum power allocation for the DF protocol for a more general multihop scenario have been further investigated in [11]. In [5], [12], and [13], an idea of distributed space-time coding has been considered by which all cooperation nodes form virtual antenna array and synchronously encode information using existing space-time codes.

Most of the works in [4]-[13] assume that the destination has perfect knowledge of channel state information (CSI) for all transmission links. While, in some scenarios, e.g., slow fading environment, the CSI is likely to be acquired by the use of pilot symbols, it may not be possible in the fast fading environment. In addition, it is questionable as to how the destination obtains source-relay channel perfectly through pilot signal forwarding without noise amplification. Moreover, the computational overhead for channel estimation increases proportional to the product of the number of transmit antennas and the number of relaying nodes.

To overcome such problems and reduce receiver complexity, various cooperative noncoherent/differential schemes [14]-[16] have been proposed. In [14], symbol error rate analysis based on the moment generating function method was provided for a specific two-hop relay systems with coherent detection and differential detection. In [15], a framework of noncoherent cooperative diversity has been proposed for the DF protocol employing frequency shift keying signals. However, the framework does not fit to the DPSK modulation. Recently, a specific two-user differential cooperation scheme was proposed in [16]. Nevertheless, the scheme relies on synchronization among users in the networks, and the scheme provides limited transmission rate.

In this paper, we propose a threshold-based differential cooperative scheme employing the DF protocol. The proposed scheme not only alleviates the above-mentioned problems of synchronization and rate limitation, but also efficiently exploits inherent spatial diversity through relay channels by the use of a predetermined decision threshold. In particular, the relay helps forward the source symbol only if the symbol is correctly decoded. At the destination, the received signal from the relay is combined with that from the source only if its amplitude is larger than the threshold; otherwise only the received signal from the source is used for the detection. A properly designed threshold allows the destination to make a judgment whether the signal from the relay contains the information such that the signals from the source and the relay can be efficiently combined and jointly decoded. We analyze bit error rate (BER) performance of the proposed threshold-based differential DF scheme employing differential M-ary phase shift keying (DMPSK) mod- ulation. We consider an ideal case where the relay is able to judge whether each decoded symbol is correct or not. The performance in this case serves as a performance benchmark, and the proposed scheme can achieve it in an ideal situation that the error propagation at the relay can be negligible. First, a tight approximate BER formulation is derived. Then, for better understanding, the proposed scheme performance, we also provide BER upper bound and BER lower bound. Based on the tight BER approximation, we jointly determine optimum decision threshold and power allocation for different scenarios. Simulation results are shown to validate our proposed scheme and support our analytical analysis.

The rest of the paper is organized as follows. Section II outlines the proposed threshold-based differential DF scheme. In Section III, we analyze BER performance and then derive a tight BER approximation of the proposed scheme under the case of perfect judgment on the decoding symbol at the relay. BER lower bound and BER upper bound of the proposed scheme are given in Section IV. In Section V, optimum threshold and optimum power allocation based on the tight BER approximation are jointly determined. Simulation results and discussions are provided in Section VI. Finally, Section VII concludes the paper.

\section{Proposed Threshold-BASEd Differential DECODE-AND-FORWARD SCHEME}

This section presents detailed descriptions of the proposed threshold-based differential scheme for DF cooperation systems. As shown in Fig. 1, we consider a two-user cooperation system in which signal transmission involves two transmission phases. A user who sends information directly to the destination is considered as a source node. The other user who helps forward the information from the source node is a relay node. In Phase I, the source differentially encodes its information and then transmits the encoded symbol to the destination. Due to the broadcasting nature of the wireless networks, the relay is also able to receive the transmitted symbol from the source. In Phase II, while the source is silent, the relay differentially decodes the received signal from the source. If the relay correctly decodes the transmitted symbol, the relay differentially re-encodes the information, and then forwards the encoded symbol to the destination. Otherwise, the relay does not send or remains idle. In both phases, we assume that all users transmit their signals through orthogonal channels by the use of existing schemes such as time division multiple access (TDMA), frequency division multiple access (FDMA), or code division multiple access (CDMA).

As will be shown in simulation results, the proposed scheme achieves higher diversity gain than the noncooperative transmission, and the obtained diversity is the same as that of the distributed space-time coding scheme [12], [13]. Compared to the distributed space-time coding scheme, the proposed scheme does not require synchronization among nodes since the signals from the source and the relay are not transmitted in the same channel. However, the bandwidth efficiency of the proposed scheme is less than that of the distributed space-time coding scheme.

In Phase I, a set of information symbols to be transmitted by the source is given by $v_{m}=e^{j \phi_{m}}$ where $\left\{\phi_{m}\right\}_{m=0}^{M-1}$ is a set of 


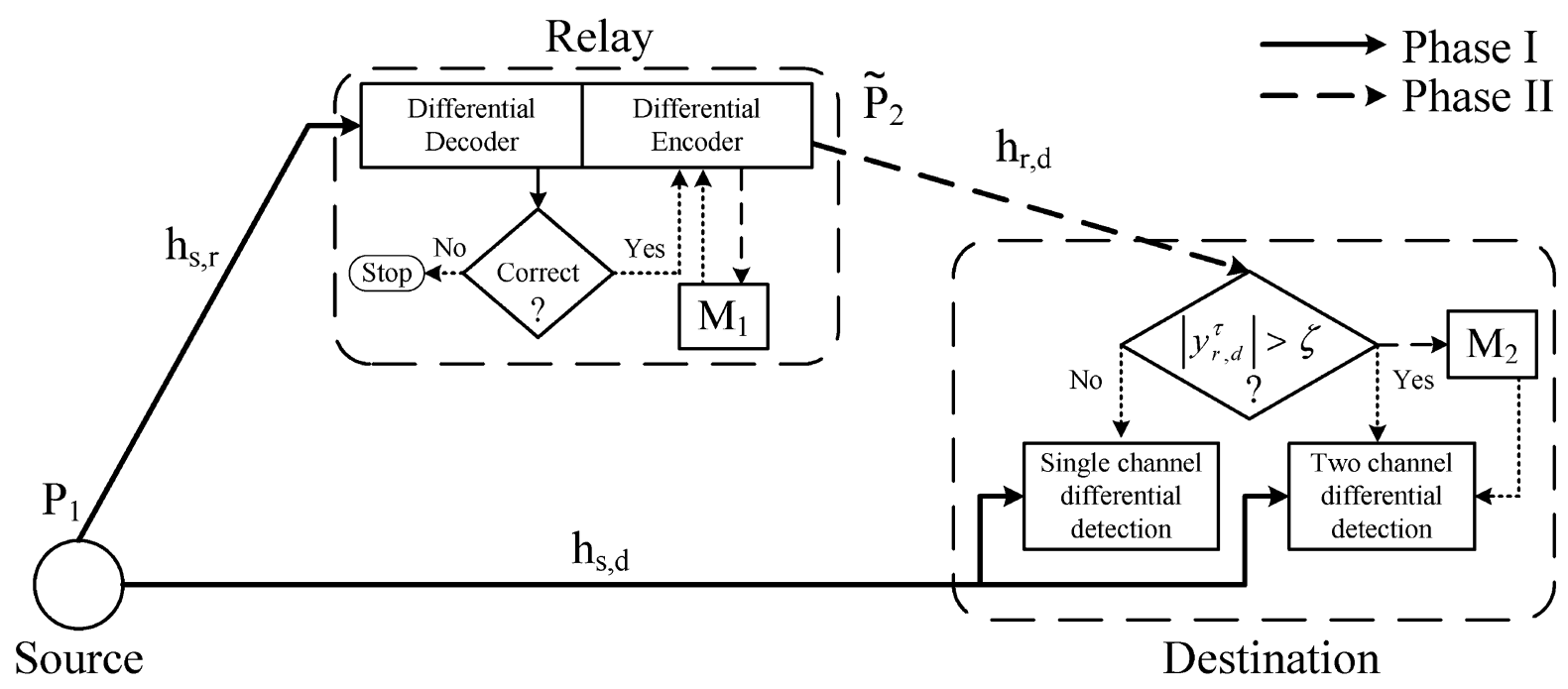

Fig. 1. Threshold-based differential scheme for decode-and-forward cooperative communications.

$M$ information phases. In the case of differential M-ary phase shift keying (DMPSK), $\phi_{m}$ is specified as $\phi_{m}=2 \pi m / M$ for $m=0,1, \ldots, M-1$. In this way, the source differentially encodes the information symbol $v_{m}$ into the phase difference between two consecutive symbols as

$$
x^{\tau}=v_{m} x^{\tau-1}
$$

where $\tau$ is the time index, and $x^{\tau}$ is the differentially encoded symbol to be transmitted at time $\tau$. After that, the source sends out $x^{\tau}$ with transmitted power $P_{1}$ to the destination and the relay. The corresponding received signals at the destination and the relay can be expressed as

$$
\begin{aligned}
& y_{s, d}^{\tau}=\sqrt{P_{1}} h_{s, d}^{\tau} x^{\tau}+w_{s, d}^{\tau} \\
& y_{s, r}^{\tau}=\sqrt{P_{1}} h_{s, r}^{\tau} x^{\tau}+w_{s, r}^{\tau}
\end{aligned}
$$

where $h_{s, d}^{\tau}$ and $h_{s, r}^{\tau}$ are fading coefficients at the source-destination link and the source-relay link, respectively, and $w_{s, d}^{\tau}$ and $w_{s, r}^{\tau}$ are additive noise. Both channel coefficients $h_{s, d}^{\tau}$ and $h_{s, r}^{\tau}$ are modeled as zero-mean complex Gaussian random variables with variances $\sigma_{s, d}^{2}$ and $\sigma_{s, r}^{2}$, i.e., $\mathcal{C N}\left(0, \sigma_{s, d}^{2}\right)$ and $\mathcal{C N}\left(0, \sigma_{s, r}^{2}\right)$, respectively. Each of the additive noise terms is modeled as $\mathcal{C N}\left(0, \mathcal{N}_{0}\right)$ where $\mathcal{N}_{0}$ represents the Gaussian noise variance.

In Phase II, the relay differentially decodes the transmitted symbol from the source. Two consecutive received signals, $y_{s, r}^{\tau-1}$ and $y_{s, r}^{\tau}$, are required to recover the transmitted information at each symbol period. By assuming that the channel coefficient $h_{s, r}^{\tau}$ is almost constant over two symbol periods, the relay differentially decodes based on the decision rule [18]

$$
\hat{m}=\arg \max _{m=0,1, \ldots, M-1} \operatorname{Re}\left\{\left(v_{m} y_{s, r}^{\tau-1}\right)^{*} y_{s, r}^{\tau}\right\}
$$

by which the CSI is not required. In the relay-cooperation mode, the relay decides whether to forward the received information or not according to the quality of the received signal. For mathematical tractability, we assume that the relay can judge whether the decoded information is correct or not. ${ }^{1}$ If the relay incorrectly decodes the received signal, such an incorrectly decoded symbol is discarded, and the relay does not send any information. Otherwise, the relay differentially re-encodes the correctly decoded information symbol and forwards it to the destination.

In conventional differential detection, successful differential decoding requires that the encoder differentially encodes each information symbol with the previously transmitted symbol. In this way, if the information symbols are sent every time slot, then the information symbol to be transmitted at time $\tau$ is differentially encoded with the transmitted symbol at time $\tau-1$. However, in the proposed differential DF scheme, the information symbols at the relay are transmitted only if they are correctly decoded. Therefore, the transmission time of the previously transmitted symbol can be any time before the current time $\tau$. We denote such previous transmission time as $\tau-k$ for $k \geq 1$, i.e., $\tau-k$ is the latest time that the relay correctly decodes the symbol before time $\tau$. In order to perform successful differential en/decoding, we let a memory $M_{1}$ at the relay (see Fig. 1) store the transmitted symbol at time $\tau-k$. Note that having a memory $M_{1}$ does not increase the system complexity compared to the conventional differential system. The difference is that the memory in our proposed scheme stores the transmitted symbol at time $\tau-k$ instead of time $\tau-1$ as does the conventional differential scheme. The differentially re-encoded signal at the relay in Phase II can be expressed as

$$
\tilde{x}^{\tau}=v_{m} \tilde{x}^{\tau-k}
$$

where $\tilde{x}^{\tau}$ is the differentially encoded symbol at the relay at time $\tau$. We can see from (1) and (5) that the differentially encoded symbols $\tilde{x}^{\tau}$ at the relay and $x^{\tau}$ at the source convey the same information symbol $v_{m}$. However, the two encoded symbols can be different since the relay differentially encodes the information symbol $v_{m}$ with the symbol in the memory which

\footnotetext{
${ }^{1}$ Practically, this can be done at the relay by applying a simple SNR threshold test to each received symbol. However, this may lead to some error propagation; the effect of error propagation on the system performance is illustrated by simulation examples in Section VI
} 
need not be $x^{\tau-1}$ as used at the source. After differential re-encoding, the relay sends the symbol $\tilde{x}^{\tau}$ to the destination with transmitted power $P_{2}$, and then stores the transmitted symbol $\tilde{x}^{\tau}$ in the memory $M_{1}$ for subsequent differential encoding. In this way, the received signal at the destination from the relay in Phase II can be expressed as

$$
y_{r, d}^{\tau}= \begin{cases}\sqrt{P_{2}} h_{r, d}^{\tau} \tilde{x}^{\tau}+w_{r, d}^{\tau}, & \text { if relay correctly decodes } \\ w_{r, d}^{\tau}, & \text { if relay incorrectly decodes }\end{cases}
$$

where $h_{r, d}^{\tau}$ is the channel coefficient from the relay to the destination and $w_{r, d}^{\tau}$ is an additive noise. We assume that $h_{r, d}^{\tau}$ is $\mathcal{C N}\left(0, \sigma_{r, d}^{2}\right)$ distributed, and $w_{r, d}^{\tau}$ is $\mathcal{C N}\left(0, \mathcal{N}_{0}\right)$ distributed.

The received signal at the destination comprises the received signal from the source in Phase I and that from the relay in Phase II. As discussed previously, in Phase II, the relay may forwards the information or remains idle. Without knowledge of the CSI, the destination is unable to know whether the received signal from the relay contains the information or not. To allow the destination make decision whether to combine the signals from the source and the relay, we propose to use a decision threshold $\zeta$ at the destination node (see Fig. 1). In this way, the received signal at the relay-destination link with amplitude $\left|y_{r, d}^{\tau}\right|$ greater than the threshold $\zeta$ is considered as a high-potential information bearing signal to be used for further differential detection.

Particularly, if the amplitude of the received signal from the relay is not greater than the decision threshold, i.e., $\left|y_{r, d}^{\tau}\right| \leq \zeta$, the destination estimates the transmitted symbol based only on the received signal from the direct link. On the other hand, if $\left|y_{r, d}^{\tau}\right|>\zeta$, the received signal from the source and that from the relay are combined together, and then the combined output is jointly differentially decoded. Note that, for successfully decoding, the differential detector requires previously received signal to serve as a CSI estimate. Since the received signal from the relay may contain the transmitted symbol or may be only noise, we propose to use a memory $M_{2}$ at the destination as shown in Fig. 1 to store the previously received signal at the relay-destination link that tends to contain the information. An ideal situation is to let the memory stores the received signal $y_{r, d}^{\tau}$ only when the signal contains the transmitted symbol; however, such information is not available since the destination does not have the knowledge of the CSI. In order to efficiently decode the received signal from the relay, the memory $M_{2}$ is used to store the received signal $y_{r, d}^{\tau}$ whose amplitude is greater than the decision threshold. If the threshold is properly designed, then the signal in the memory $M_{2}$ corresponds to the received signal from the relay that carries the encoded symbol stored in the memory $M_{1}$. With an assumption that the channel coefficients stay almost constant for several symbol periods, the signal in the memory $M_{2}$ serves as a channel estimate of the relay-destination link and it is used for efficient differential decoding at the destination. Based on the multichannel differential detection in [1], the combined signal before being differentially decoded is

$$
y= \begin{cases}a_{1}\left(y_{s, d}^{\tau-1}\right)^{*} y_{s, d}^{\tau}+a_{2}\left(y_{r, d}^{\tau-l}\right)^{*} y_{r, d}^{\tau}, & \text { if }\left|y_{r, d}^{\tau}\right|>\zeta \\ \left(y_{s, d}^{\tau-1}\right)^{*} y_{s, d}^{\tau}, & \text { if }\left|y_{r, d}^{\tau}\right| \leq \zeta\end{cases}
$$

where $a_{1}$ and $a_{2}$ are combining weights, and $\tau-l(l \geq 1)$ represents the time index of the latest signal in memory $M_{2}$, i.e., $y_{r, d}^{\tau-l}$ is the most recent received signal from the relay whose amplitude is larger than the threshold. Note that different combining weights, $a_{1}$ and $a_{2}$, result in different system performances. In Section III, we determine the BER performance in the case of $a_{1}=\left(1 / 2 \mathcal{N}_{0}\right)$ and $a_{2}=\left(1 / 2 \mathcal{N}_{0}\right)$; these two combining weights maximize the signal-to-noise ratio (SNR) of the combiner output when the destination is able to differentially decode the signals from both source and relay. Although, these combining weights are not optimum in general, they are optimum for a case that signals from both source-destination and relay-destination links contain information and the corresponding channel variances for these two links are the same. Based on the combined signal in (7), the decoder at the destination jointly differentially decodes the transmitted information symbol by using the following decision rule:

$$
\hat{m}=\arg \max _{m=0,1, \ldots, M-1} \operatorname{Re}\left\{v_{m}^{*} y\right\} .
$$

\section{BER ANALYSIS}

In this section, we analyze the BER performance of the proposed threshold-based differential DF scheme employing DMPSK modulation. We focus on an ideal case when the relay is able to judge whether each decoded symbol is correct. This leads to a performance benchmark, and the proposed scheme can achieve it in an ideal situation that the error propagation at the relay can be negligible. First, we classify different scenarios that correspond to different instantaneous SNRs at the combiner output of the destination. Next, the probability that each scenario occurs is determined. Then, we evaluate an average BER performance by taking into account all the possible scenarios.

\section{A. Classification of Different Scenarios}

We classify in this section all different scenarios that result in different SNRs at the combiner output. Recall that, if the amplitude of received signal from the relay is larger than the decision threshold $\left(\left|y_{r, d}^{\tau}\right|>\zeta\right)$, then the destination jointly decodes the received signals from the source $\left(y_{s, d}^{\tau}\right)$ and the relay $\left(y_{r, d}^{\tau}\right)$. Otherwise, only the received signal $y_{s, d}^{\tau}$ from the source is used for differential detection. Therefore, we can classify the scenarios into two major groups, namely the scenarios that $\left|y_{r, d}^{\tau}\right|>\zeta$ and $\left|y_{r, d}^{\tau}\right| \leq \zeta$. In the case that $\left|y_{r, d}^{\tau}\right| \leq \zeta$, the SNR can be simply determined based on the received signal from the direct link. On the other hand, if $\left|y_{r, d}^{\tau}\right|>\zeta$, the SNR at the combiner output depends not only on the received signals from the direct link but also on that from the relay link. According to the decision rule in (7), if $\left|y_{r, d}^{\tau}\right|>\zeta$, the performance of the differential decoder relies on the received signals from the source at the current time $\tau\left(y_{s, d}^{\tau}\right)$ and the previous time $\tau-1\left(y_{s, d}^{\tau-1}\right)$ as well as the received signal from the relay at the current time $\tau\left(y_{r, d}^{\tau}\right)$ and that stored in the memory $\left(y_{r, d}^{\tau-l}\right)$. The received signals $y_{r, d}^{\tau}$ and $y_{r, d}^{\tau-l}$ from the relay may or may not contain the information, depending on the correctness of the decoded symbol at the relay at time $\tau$ and time $\tau-l$. If the relay decodes the symbol correctly, then the relay sends the encoded symbol with transmitted power $P_{2}$, otherwise, the transmitted power is zero. Thus, based on the transmitted power at the relay at time $\tau$ and time $\tau-l$, 


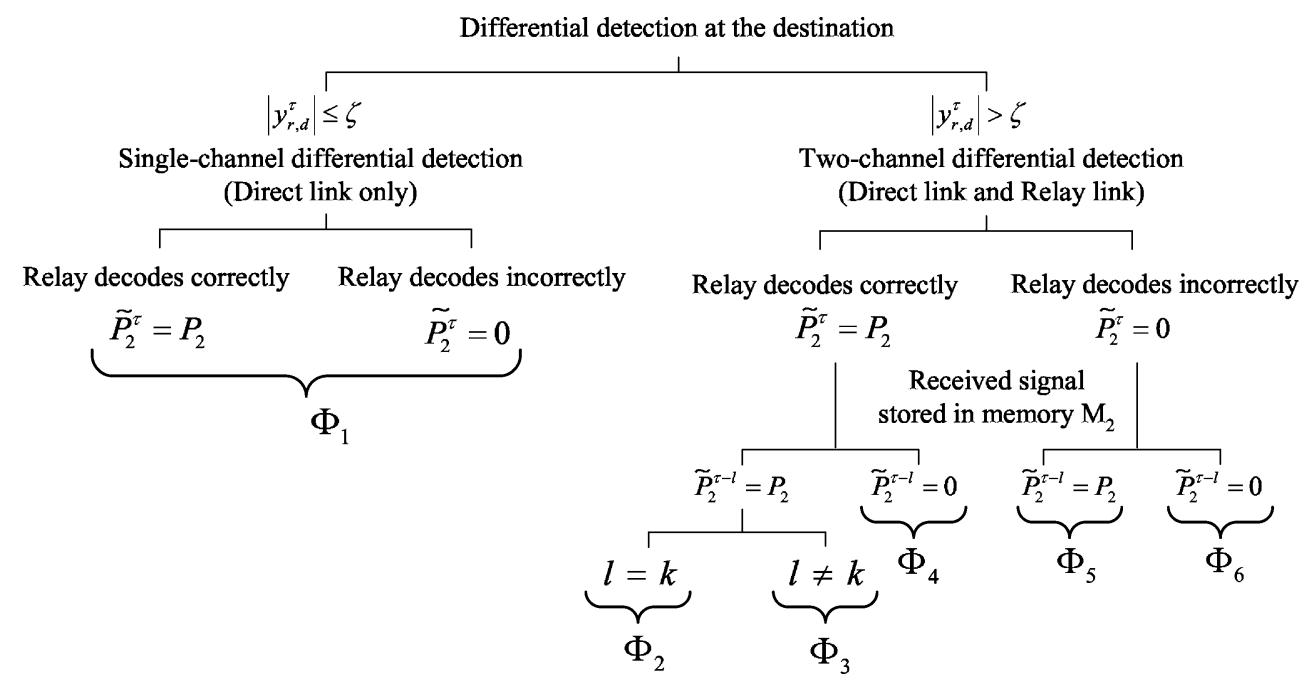

Fig. 2. Two possible differential detection techniques at the destination; single-channel differential detection or two-channel differential detection, with six possible scenarios based on the currently received signal and the signal stored in memory $M_{2}$.

we can further classify the scenarios into four different categories, namely (a) $\tilde{P}_{2}^{\tau}=P_{2}$ and $\tilde{P}_{2}^{\tau-l}=P_{2}$, (b) $\tilde{P}_{2}^{\tau}=P_{2}$ and $\tilde{P}_{2}^{\tau-l}=0$, (c) $\tilde{P}_{2}^{\tau}=0$ and $\tilde{P}_{2}^{\tau-l} \stackrel{2}{=} P_{2}$, as well as (d) $\tilde{P}_{2}^{\tau}=0$ and $\tilde{P}_{2}^{\tau-l}=0$. In the case that $\tilde{P}_{2}^{\tau}=P_{2}$ and $\tilde{P}_{2}^{\tau-l}=P_{2}$, the received signals $y_{r, d}^{\tau}$ and $y_{r, d}^{\tau-l}$ convey the symbols $\tilde{x}^{\tau}$ and $\tilde{x}^{\tau-l}$, respectively. Since the relay differentially encodes the information symbol from the source with the symbol $\tilde{x}^{\tau-k}$ in the memory $M_{1}$, the SNR at the combiner output also depends on whether the received signal $y_{r, d}^{\tau-l}$ used for decoding corresponds to the symbol $\tilde{x}^{\tau-k}$ used for encoding. Therefore, the scenarios under $\tilde{P}_{2}^{\tau}=P_{2}$ and $\tilde{P}_{2}^{\tau-l}=P_{2}$ can be further separated into two cases, namely $l=k$ and $l \neq k$. All of these six possible scenarios are summarized in Fig. 2.

In order to facilitate the BER analysis in the subsequent section, we define six different scenarios by $\Phi_{i}, i=1,2, \ldots, 6$ as follows. We let the first scenario $\Phi_{1}$ be the scenario that the amplitude of the received signal is not larger than the threshold $\zeta$, i.e.,

$$
\Phi_{1} \triangleq\left\{\left|y_{r, d}^{\tau}\right| \leq \zeta\right\} .
$$

When $\Phi_{1}$ occurs, the destination does not combine the received signal from the relay with that from the source. The second scenario $\Phi_{2}$ is a scenario that consists of three joint events, namely the amplitude of the received signal is larger than the threshold, the relay transmitted powers at both time $\tau$ and $\tau-l$ are equal to $P_{2}$, and the received signal $y_{r, d}^{\tau-l}$ stored in memory $M_{2}$ conveys the information symbol $\tilde{x}^{\tau-k}$ stored in memory $M_{1}$. Specifically, $\Phi_{2}$ can be written as

$$
\Phi_{2} \triangleq\left\{\left|y_{r, d}^{\tau}\right|>\zeta, \tilde{P}_{2}^{\tau}=P_{2}, \tilde{P}_{2}^{\tau-l}=P_{2}, l=k\right\} .
$$

The scenario $\Phi_{3}$ is similar to the scenario $\Phi_{2}$ excepts that the received signal $y_{r, d}^{\tau-l}$ does not contain the symbol $\tilde{x}^{\tau-k}$, i.e., $l \neq k$. We express this scenario as $\Phi_{3} \triangleq\left\{\left|y_{r, d}^{\tau}\right|>\zeta, \tilde{P}_{2}^{\tau}=\right.$ $\left.P_{2}, \tilde{P}_{2}^{\tau-l}=P_{2}, l \neq k\right\}$. The scenarios $\Phi_{4}$ to $\Phi_{6}$ correspond to the scenarios that $\left|y_{r, d}^{\tau}\right|>\zeta$ and either $\tilde{P}_{2}^{\tau}$ or $\tilde{P}_{2}^{\tau-l}$ is zero. Under the scenario $\Phi_{4}$, the transmitted power $\tilde{P}_{2}^{\tau}$ is $P_{2}$ whereas the transmitted power $\tilde{P}_{2}^{\tau-l}$ is 0 . We express the scenario $\Phi_{4}$ as $\Phi_{4} \triangleq\left\{\left|y_{r, d}^{\tau}\right|>\zeta, \tilde{P}_{2}^{\tau}=P_{2}, \tilde{P}_{2}^{\tau-l}=0\right\}$. Under the scenario $\Phi_{5}$, the transmitted power $\tilde{P}_{2}^{\tau}$ is 0 whereas the transmitted power $\tilde{P}_{2}^{\tau-l}$ is $P_{2}$, i.e., $\Phi_{5} \triangleq\left\{\left|y_{r d}^{\tau}\right|>\zeta, \tilde{P}_{2}^{\tau}=0, \tilde{P}_{2}^{\tau-l}=\right.$ $\left.P_{2}\right\}$. The scenario $\Phi_{6}$ corresponds to the scenario that both $\tilde{P}_{2}^{\tau}$ and $\tilde{P}_{2}^{\tau-l}$ are zeros. We define the scenario $\Phi_{6}$ as $\Phi_{6} \triangleq$ $\left\{\left|y_{r, d}^{\tau}\right|>\zeta, \tilde{P}_{2}^{\tau}=0, \tilde{P}_{2}^{\tau-l}=0\right\}$.

For subsequent derivations, we denote $\left.P_{\mathrm{BER}}^{h}\right|_{i}$ as the conditional BER given a scenario $\Phi_{i}$ and a set of channel realizations including the source-destination link, the source-relay link, and the relay-destination link. We also denote $P_{r}^{h}\left(\Phi_{i}\right)$ as the chance that the scenario $\Phi_{i}$ occurs given a set of channel realizations. Accordingly, the conditional BER of the proposed differential DF scheme can be expressed as

$$
P_{\mathrm{BER}}^{h}=\left.\sum_{i=1}^{6} P_{\mathrm{BER}}^{h}\right|_{\Phi_{i}} P_{r}^{h}\left(\Phi_{i}\right) .
$$

By averaging (11) over all channel realizations, the average BER of the proposed scheme is given by

$$
P_{\mathrm{BER}}=\sum_{i=1}^{6} \mathrm{E}\left[\left.P_{\mathrm{BER}}^{h}\right|_{\Phi_{i}} P_{r}^{h}\left(\Phi_{i}\right)\right]=\sum_{i=1}^{6} P_{\mathrm{BER}}^{(i)}
$$

where $\mathrm{E}[\cdot]$ represents the expectation operation.

In the following sections, we determine the chance that each scenario occurs $\left(P_{r}^{h}\left(\Phi_{i}\right)\right)$, the conditional BER $\left(\left.P_{\mathrm{BER}}^{h}\right|_{\Phi_{i}}\right)$, and finally obtain the average BER $\left(P_{\mathrm{BER}}\right)$ of the proposed differential DF scheme.

\section{B. Probability of Occurrence}

To determine the chance that each scenario $\Phi_{i}$ occurs, we first note that the amplitude of the received signal $y_{r, d}^{\tau}$ depends on the relay transmitted power $\tilde{P}_{2}^{\tau}$ or the correctness of the decoded symbol at the relay. With DMPSK signals, the chance of incorrect decoding at the relay, i.e., $\tilde{P}_{2}^{\tau}=0$, can be obtained from the conditional SER [17] as

$$
\begin{aligned}
P_{r}^{h}\left(\tilde{P}_{2}^{\tau}=0\right) & =\frac{1}{\pi} \int_{0}^{(M-1) \pi / M} \exp \left[-g(\theta) \gamma_{s, r}^{\tau}\right] d \theta \\
& \triangleq \Psi\left(\gamma_{s, r}^{\tau}\right)
\end{aligned}
$$


where

$$
\gamma_{s, r}^{\tau}=\frac{P_{1}\left|h_{s, r}^{\tau}\right|^{2}}{\mathcal{N}_{0}}
$$

is the instantaneous SNR per symbol at the relay due to the transmitted symbol from the source, and

$$
g(\theta)=\frac{\sin ^{2}(\pi / M)}{1+\cos (\pi / M) \cos (\theta)} .
$$

Similarly, the chance that the relay forwards the symbol with transmitted power $\tilde{P}_{2}^{\tau}=P_{2}$ is determined by the chance of correct decoding at the relay, hence

$$
P_{r}^{h}\left(\tilde{P}_{2}^{\tau}=P_{2}\right)=1-\Psi\left(\gamma_{s, r}^{\tau}\right)
$$

where $\Psi\left(\gamma_{s, r}^{\tau}\right)$ is specified in (13).

Consider the scenario $\Phi_{1}$ in which the amplitude of the received signal $y_{r, d}^{\tau}$ is not greater than the decision threshold. By using (13)-(16), the chance that $\Phi_{1}$ occurs can be written as

$$
\begin{aligned}
P_{r}^{h}\left(\Phi_{1}\right)= & P_{r}^{h}\left(\left|y_{r, d}^{\tau}\right| \leq \zeta \mid \tilde{P}_{2}^{\tau}=0\right) \Psi\left(\gamma_{s, r}^{\tau}\right) \\
& +P_{r}^{h}\left(\left|y_{r, d}^{\tau}\right| \leq \zeta \mid \tilde{P}_{2}^{\tau}=P_{2}\right)\left[1-\Psi\left(\gamma_{s, r}^{\tau}\right)\right]
\end{aligned}
$$

The conditional probabilities, $P_{r}^{h}\left(\left|y_{r, d}^{\tau}\right| \leq \zeta \mid \tilde{P}_{2}^{\tau}=0\right)$ and $P_{r}^{h}\left(\left|y_{r, d}^{\tau}\right| \leq \zeta \mid \tilde{P}_{2}^{\tau}=P_{2}\right)$ can be obtained from the cumulative distribution function (CDF) of the random variable $\left|y_{r, d}^{\tau}\right|$. The received signal $y_{r, d}^{\tau}$ is a complex Gaussian random variable with mean $\sqrt{\tilde{P}_{2}} h_{r, d}^{\tau} \tilde{x}^{\tau}$ and variance $\mathcal{N}_{0}$, i.e., $y_{r, d}^{\tau} \sim \mathcal{C N}\left(\sqrt{\tilde{P}_{2}} h_{r, d}^{\tau} \tilde{x}^{\tau}, \mathcal{N}_{0}\right)$. If $\tilde{P}_{2}^{\tau}=0$ which results from incorrect decoding at the relay, the received signal $y_{r, d}^{\tau}$ is a zero-mean complex Gaussian random variable with variance $\mathcal{N}_{0}\left(y_{r, d}^{\tau} \sim \mathcal{C N}\left(0, \mathcal{N}_{0}\right)\right)$, and its amplitude is Rayleigh distributed. Hence, the conditional probability that $\left|y_{r, d}^{\tau}\right|$ is not greater than the decision threshold given that the relay does not send information can be expressed as [1]

$$
P_{r}^{h}\left(\left|y_{r, d}^{\tau}\right| \leq \zeta \mid \tilde{P}_{2}^{\tau}=0\right)=1-\exp \left(-\zeta^{2} / \mathcal{N}_{0}\right)
$$

If $\tilde{P}_{2}^{\tau}=P_{2}$ which corresponds to the case of correct decoding at the relay, then $y_{r, d}^{\tau}$ is Gaussian distributed with mean $\sqrt{P_{2}} h_{r, d}^{\tau} \tilde{x}^{\tau}$ and variance $\mathcal{N}_{0}$. In this case, $\left|y_{r, d}^{\tau}\right|$ is a Ricean-distributed random variable. The conditional probability that $\left|y_{r, d}^{\tau}\right|$ is not greater than the decision threshold, given that the relay sends the information with transmitted power $P_{2}$, can be obtained based on the results in [1] as

$P_{r}^{h}\left(\left|y_{r, d}^{\tau}\right| \leq \zeta \mid \tilde{P}_{2}^{\tau}=P_{2}\right)=1-\mathcal{M}\left(P_{2}\left|h_{r, d}^{\tau}\right|^{2}, \zeta\right)$

where

$\mathcal{M}\left(P_{2}\left|h_{r, d}^{\tau}\right|^{2}, \zeta\right) \triangleq Q_{1}\left(\sqrt{\frac{P_{2}\left|h_{r, d}^{\tau}\right|^{2}}{\mathcal{N}_{0} / 2}}, \frac{\zeta}{\sqrt{\mathcal{N}_{0} / 2}}\right)$

in which $Q_{1}(\alpha, \beta)$ is the Marcum $Q$-function [18]

$$
Q_{1}(\alpha, \beta)=\int_{\beta}^{\infty} \lambda \exp \left[-\left(\frac{\lambda^{2}+\alpha^{2}}{2}\right)\right] I_{0}(\alpha \lambda) d \lambda
$$

and $I_{0}(\cdot)$ is the zeroth-order modified Bessel function of the first kind. By substituting (18) and (19) into (17), we can express the chance that $\Phi_{1}$ occurs as

$$
\begin{aligned}
P_{r}^{h}\left(\Phi_{1}\right)= & \left(1-\exp \left(-\zeta^{2} / \mathcal{N}_{0}\right)\right) \Psi\left(\gamma_{s, r}^{\tau}\right) \\
& +\left(1-\mathcal{M}\left(P_{2}\left|h_{r, d}^{\tau}\right|^{2}, \zeta\right)\right)\left[1-\Psi\left(\gamma_{s, r}^{\tau}\right)\right] .
\end{aligned}
$$

The rest of the scenarios, $\Phi_{2}$ to $\Phi_{6}$, are related to the situation when the amplitude of the received signal from the relay, $\left|y_{r, d}^{\tau}\right|$, is greater than the decision threshold $\zeta$. In these scenarios, both the currently received signal $\left(y_{r, d}^{\tau}\right)$ and that stored in the memory $M_{2}\left(y_{r, d}^{\tau-l}\right)$ are used for differential detection at the destination. In the proposed scheme, the memory $M_{2}$ stores only the received signal from the relay whose amplitude is larger than the threshold. This implies that the amplitude $\left|y_{r, d}^{\tau-l}\right|$ is larger than the threshold. Therefore, the chance that each of the scenarios $\Phi_{2}$ to $\Phi_{6}$ happens is conditioned on the event that $\left|y_{r, d}^{\tau-l}\right|>\zeta$. From (10), the chance that the scenario $\Phi_{2}$ occurs is given by

$$
\begin{aligned}
P_{r}^{h}\left(\Phi_{2}\right)=P_{r}^{h}\left(\left|y_{r, d}^{\tau}\right|>\zeta, \tilde{P}_{2}^{\tau}=P_{2},\right. & \\
& \left.\tilde{P}_{2}^{\tau-l}=P_{2}, l=k|| y_{r, d}^{\tau-l} \mid>\zeta\right) .
\end{aligned}
$$

Since the events at time $\tau-l$ are independent of the events at time $\tau, P_{r}^{h}\left(\Phi_{2}\right)$ can be written as a product of the probabilities

$$
\begin{aligned}
P_{r}^{h}\left(\Phi_{2}\right)=P_{r}^{h} & \left(\left|y_{r, d}^{\tau}\right|>\zeta, \tilde{P}_{2}^{\tau}=P_{2}\right) \\
& \times P_{r}^{h}\left(\tilde{P}_{2}^{\tau-l}=P_{2}, l=k|| y_{r, d}^{\tau-l} \mid>\zeta\right) .
\end{aligned}
$$

The first term on the right hand side of (24) represents the probability that the relay transmits the decoded symbol with power $P_{2}$ and the received signal from the relay is larger than the threshold. This term can be reexpressed as

$$
\begin{aligned}
& P_{r}^{h}\left(\left|y_{r, d}^{\tau}\right|>\zeta, \tilde{P}_{2}^{\tau}=P_{2}\right)=P_{r}^{h}\left(\left|y_{r, d}^{\tau}\right|>\zeta \mid \tilde{P}_{2}^{\tau}=P_{2}\right) \\
& \quad \times P_{r}^{h}\left(\tilde{P}_{2}^{\tau}=P_{2}\right) .
\end{aligned}
$$

In (25), the chance that the amplitude of the received signal from the relay is larger than the threshold given that the relay sends the information can be obtained from (19) as

$$
P_{r}^{h}\left(\left|y_{r, d}^{\tau}\right|>\zeta \mid \tilde{P}_{2}^{\tau}=P_{2}\right)=\mathcal{M}\left(P_{2}\left|h_{r, d}^{\tau}\right|^{2}, \zeta\right) .
$$

Therefore, using the results in (16) and (26), (25) can be rewritten as

$$
P_{r}^{h}\left(\left|y_{r, d}^{\tau}\right|>\zeta, \tilde{P}_{2}^{\tau}=P_{2}\right)=\mathcal{M}\left(P_{2}\left|h_{r, d}^{\tau}\right|^{2}, \zeta\right)\left(1-\Psi\left(\gamma_{s, r}^{\tau}\right)\right)
$$

Next, we consider the second term on the right hand side of (24) which represents the chance that the relay transmits with power $\tilde{P}_{2}^{\tau-l}=P_{2}$, and the received signal $y_{r, d}^{\tau-l}$ stored in the memory $M_{2}$ conveys the information symbol $\tilde{x}^{\tau-k}$ stored in the memory $M_{1}$. By using the concept of conditional probability [20], we can find that

$$
\begin{aligned}
P_{r}^{h} & \left(\tilde{P}_{2}^{\tau-l}=P_{2}, l=k|| y_{r, d}^{\tau-l} \mid>\zeta\right) \\
\quad= & \sum_{k \geq 1} P_{r}^{h}\left(\tilde{P}_{2}^{\tau-k}=P_{2}|| y_{r, d}^{\tau-k} \mid>\zeta\right) P_{r}^{h}\left(\left|y_{r, d}^{\tau-k}\right|>\zeta\right)
\end{aligned}
$$




$$
\times \prod_{i=1}^{k-1} P_{r}^{h}\left(\left|y_{r, d}^{\tau-i}\right| \leq \zeta\right) P_{r}^{h}\left(\tilde{P}_{2}^{\tau-i}=0|| y_{r, d}^{\tau-i} \mid \leq \zeta\right)
$$

By using the result in (27), the term $P_{r}^{h}\left(\tilde{P}_{2}^{\tau-k}=\right.$ $\left.P_{2}|| y_{r, d}^{\tau-k} \mid>\zeta\right) P_{r}^{h}\left(\left|y_{r, d}^{\tau-k}\right|>\zeta\right)$ in (28) can be evaluated as $\mathcal{M}\left(P_{2}\left|h_{r, d}^{\tau-k}\right|^{2}, \zeta\right)\left(1-\Psi\left(\gamma_{s, r}^{\tau-k}\right)\right)$. If the channels stay almost constant for several time slots, then it can be further approximated by $\mathcal{M}\left(P_{2}\left|h_{r, d}^{\tau}\right|^{2}, \zeta\right)\left(1-\Psi\left(\gamma_{s, r}^{\tau}\right)\right)$. Note that the derivation in (28) assumes an infinite packet size; however, the impact of packet size on the performance is negligible because the error propagation stops whenever one symbol is correctly decoded at the relay and the destination. In addition, a chance of having large $k$ is very small, as it corresponds to the case when all $k-1$ consecutive symbols are incorrectly decoded at the relay. By applying Bayes' rule [20] and using the results in (13) and (18), we can express the product term in (28) as

$$
\begin{aligned}
& \prod_{i=1}^{k-1} P_{r}^{h}\left(\left|y_{r, d}^{\tau-i}\right| \leq \zeta\right) P_{r}^{h}\left(\tilde{P}_{2}^{\tau-i}=0|| y_{r, d}^{\tau-i} \mid \leq \zeta\right) \\
& \quad=\prod_{i=1}^{k-1} P_{r}^{h}\left(\left|y_{r, d}^{\tau-i}\right| \leq \zeta \mid \tilde{P}_{2}^{\tau-i}=0\right) P_{r}^{h}\left(\tilde{P}_{2}^{\tau-i}=0\right) \\
& \quad \approx\left[\left(1-e^{-\zeta^{2} / \mathcal{N}_{0}}\right) \Psi\left(\gamma_{s, r}^{\tau}\right)\right]^{k-1}
\end{aligned}
$$

where the resulting approximation comes from approximating $P_{r}^{h}\left(\tilde{P}_{2}^{\tau-i}=0\right)=\Psi\left(\gamma_{s, r}^{\tau-i}\right)$ by $\Psi\left(\gamma_{s, r}^{\tau}\right)$ for all $i$. Accordingly, we can approximate (28) as

$$
\begin{aligned}
& P_{r}^{h}\left(\tilde{P}_{2}^{\tau-l}=P_{2}, l=k|| y_{r, d}^{\tau-l} \mid>\zeta\right) \\
& \approx \mathcal{M}\left(P_{2}\left|h_{r, d}^{\tau}\right|^{2}, \zeta\right)\left(1-\Psi\left(\gamma_{s, r}^{\tau}\right)\right) \\
& \quad \times \sum_{k \geq 1}\left[\left(1-e^{-\zeta^{2} / \mathcal{N}_{0}}\right) \Psi\left(\gamma_{s, r}^{\tau}\right)\right]^{k-1} \\
&=\frac{\mathcal{M}\left(P_{2}\left|h_{r, d}^{\tau}\right|^{2}, \zeta\right)\left(1-\Psi\left(\gamma_{s, r}^{\tau}\right)\right)}{1-\left(1-e^{\frac{-\zeta^{2}}{N_{0}}}\right) \Psi\left(\gamma_{s, r}^{\tau}\right)} .
\end{aligned}
$$

Hence, by substituting (27) and (30) into (24), the chance that the scenario $\Phi_{2}$ happens can be approximated by

$$
P_{r}^{h}\left(\Phi_{2}\right) \approx \frac{\mathcal{M}^{2}\left(P_{2}\left|h_{r, d}^{\tau}\right|^{2}, \zeta\right)\left(1-\Psi\left(\gamma_{s, r}^{\tau}\right)\right)^{2}}{1-\left(1-e^{-\zeta^{2} / \mathcal{N}_{0}}\right) \Psi\left(\gamma_{s, r}^{\tau}\right)} .
$$

Next, we consider the scenario $\Phi_{3}$ which is similar to the scenario $\Phi_{2}$ except that the received signal $y_{r, d}^{\tau-l}$ stored in the memory $M_{2}$ at the destination does not convey the symbol $\tilde{x}^{\tau-k}$ stored in the memory $M_{1}$ at the relay. The chance that the scenario $\Phi_{3}$ happens is given by

$$
\begin{aligned}
P_{r}^{h}\left(\Phi_{3}\right)=P_{r}^{h}\left(\left|y_{r, d}^{\tau}\right|>\zeta, \tilde{P}_{2}^{\tau}=P_{2}\right. \\
\left.\tilde{P}_{2}^{\tau-l}=P_{2}, l \neq k|| y_{r, d}^{\tau-l} \mid>\zeta\right) .
\end{aligned}
$$

Observe that the scenarios $\Phi_{2}$ and $\Phi_{3}$ are disjoint. Thus, the chance that the scenario $\Phi_{3}$ happens can be obtained from $P_{r}^{h}\left(\Phi_{2}\right)$ as

$$
P_{r}^{h}\left(\Phi_{3}\right)=P_{r}^{h}\left(\Phi_{2} \cup \Phi_{3}\right)-P_{r}^{h}\left(\Phi_{2}\right)
$$

where $P_{r}^{h}\left(\Phi_{2} \cup \Phi_{3}\right)=P_{r}^{h}\left(\left|y_{r, d}^{\tau}\right|>\zeta, \tilde{P}_{2}^{\tau}=P_{2}, \tilde{P}_{2}^{\tau-l}=\right.$ $\left.P_{2},|| y_{r, d}^{\tau-l} \mid>\zeta\right)$. Since the signals at time $\tau$ and time $\tau-l$ are independent, we can express $P_{r}^{h}\left(\Phi_{2} \cup \Phi_{3}\right)$ as

$$
\begin{aligned}
P_{r}^{h}\left(\Phi_{2} \cup \Phi_{3}\right)=P_{r}^{h} & \left(\left|y_{r, d}^{\tau}\right|>\zeta, \tilde{P}_{2}^{\tau}=P_{2}\right) \\
& \times P_{r}^{h}\left(\tilde{P}_{2}^{\tau-l}=P_{2}|| y_{r, d}^{\tau-l} \mid>\zeta\right) .
\end{aligned}
$$

By applying Bayes' rule, the second term on the right hand side of (34) is given by

$$
\begin{aligned}
P_{r}^{h}\left(\tilde{P}_{2}^{\tau-l}=P_{2}|| y_{r, d}^{\tau-l} \mid\right. & >\zeta) \\
& =\frac{P_{r}^{h}\left(\left|y_{r, d}^{\tau-l}\right|>\zeta, \tilde{P}_{2}^{\tau-l}=P_{2}\right)}{P_{r}^{h}\left(\left|y_{r, d}^{\tau-l}\right|>\zeta\right)} .
\end{aligned}
$$

The numerator on the right hand side of (35) is in the same form as (27) with $\tau$ replaced by $\tau-l$, whereas the denominator can be calculated by using the concept of total probability [20]

$$
\begin{aligned}
& P\left(\left|y_{r, d}^{\tau-l}\right|>\zeta\right) \\
& \quad=P\left(\left|y_{r, d}^{\tau-l}\right|>\zeta \mid \tilde{P}_{2}^{\tau-l}=P_{2}\right) P\left(\tilde{P}_{2}^{\tau-l}=P_{2}\right) \\
& \quad+P\left(\left|y_{r, d}^{\tau-l}\right|>\zeta \mid \tilde{P}_{2}^{\tau-l}=0\right) P\left(\tilde{P}_{2}^{\tau-l}=0\right) .
\end{aligned}
$$

In (36), the chance that the received signal $\left|y_{r, d}^{\tau-l}\right|$ is greater than the decision threshold given that the relay does not send information can be obtained from (18) as

$$
P_{r}^{h}\left(\left|y_{r, d}^{\tau-l}\right|>\zeta \mid \tilde{P}_{2}^{\tau}=0\right)=\exp \left(-\zeta^{2} / \mathcal{N}_{0}\right) .
$$

Substitute (13), (27), and (37) into (36) resulting in

$$
\begin{aligned}
P( & \left(\left|y_{r, d}^{\tau-l}\right|>\zeta\right) \\
= & \mathcal{M}\left(P_{2}\left|h_{r, d}^{\tau-l}\right|^{2}, \zeta\right)\left(1-\Psi\left(\gamma_{s, r}^{\tau-l}\right)\right) \\
& +\exp \left(-\zeta^{2} / \mathcal{N}_{0}\right) \Psi\left(\gamma_{s, r}^{\tau-l}\right) \\
\triangleq & \Gamma\left(P_{1}\left|h_{s, r}^{\tau-l}\right|^{2}, P_{2}\left|h_{r, d}^{\tau-l}\right|^{2}\right) .
\end{aligned}
$$

By assuming that the channel coefficients $h_{i, j}^{\tau-l} \approx h_{i, j}^{\tau}$ for any transmission link from $i$ to $j$, then from (27), (36), and (38), we can determine the chance $P_{r}^{h}\left(\Phi_{2} \cup \Phi_{3}\right)$ in (34) as

$$
P_{r}^{h}\left(\Phi_{2} \cup \Phi_{3}\right)=\frac{\mathcal{M}^{2}\left(P_{2}\left|h_{r, d}^{\tau}\right|^{2}, \zeta\right)\left(1-\Psi\left(\gamma_{s, r}^{\tau}\right)\right)^{2}}{\Gamma\left(P_{1}\left|h_{s, r}^{\tau}\right|^{2}, P_{2}\left|h_{r, d}^{\tau}\right|^{2}\right)} .
$$

Now the chance that the scenario $\Phi_{3}$ happens can be obtained by substituting (31) and (39) into (33). We can express the probability $P_{r}^{h}\left(\Phi_{3}\right)$ as

$$
\begin{aligned}
P_{r}^{h}\left(\Phi_{3}\right) & =\mathcal{M}^{2}\left(P_{2}\left|h_{r, d}^{\tau}\right|^{2}, \zeta\right)\left(1-\Psi\left(\gamma_{s, r}^{\tau}\right)\right)^{2} \\
\times & \left(\frac{1}{\Gamma\left(P_{1}\left|h_{s, r}^{\tau}\right|^{2}, P_{2}\left|h_{r, d}^{\tau}\right|^{2}\right)}\right.
\end{aligned}
$$




$$
\left.-\frac{1}{1-\left(1-e^{-\zeta^{2} / \mathcal{N}_{0}}\right) \Psi\left(\gamma_{s, r}^{\tau}\right)}\right)
$$

Following the same steps as used to determine $P_{r}^{h}\left(\Phi_{2} \cup \Phi_{3}\right)$ in (34)-(38), the chance that the scenario $\Phi_{4}$ occurs can be expressed as

$$
\begin{aligned}
& P_{r}^{h}\left(\Phi_{4}\right) \\
& =P_{r}^{h}\left(\left|y_{r, d}^{\tau}\right|>\zeta, \tilde{P}_{2}^{\tau}=P_{2}, \tilde{P}_{2}^{\tau-l}=0,|| y_{r, d}^{\tau-l} \mid>\zeta\right) \\
& =\frac{P_{r}^{h}\left(\left|y_{r, d}^{\tau}\right|>\zeta, \tilde{P}_{2}^{\tau}=P_{2}\right) P_{r}^{h}\left(\left|y_{r, d}^{\tau-l}\right|>\zeta, \tilde{P}_{2}^{\tau-l}=0\right)}{P_{r}^{h}\left(\left|y_{r, d}^{\tau-l}\right|>\zeta\right)} .
\end{aligned}
$$

By substituting (27) and (38) into (41), we arrive at (42), shown at the bottom of the page. Similarly, the chance that the scenario $\Phi_{5}$ happens can be determined as (43), shown at the bottom of the page. With an assumption that the channels at time $\tau$ and time $\tau-l$ are almost the same, we can see from (41) and (43) that the chances that the scenarios $\Phi_{4}$ and $\Phi_{5}$ happen are equal. Therefore, $P_{r}^{h}\left(\Phi_{5}\right)$ can be expressed in the same form as (42). Last, the chance that the scenario $\Phi_{6}$ occurs can be determined as

$$
\begin{array}{r}
P_{r}^{h}\left(\Phi_{6}\right)=P_{r}^{h}\left(\left|y_{r, d}^{\tau}\right|>\zeta, \tilde{P}_{2}^{\tau}=0, \tilde{P}_{2}^{\tau-l}=0,|| y_{r, d}^{\tau-l} \mid>\zeta\right) \\
=\frac{P_{r}^{h}\left(\left|y_{r, d}^{\tau}\right|>\zeta, \tilde{P}_{2}^{\tau}=0\right) P_{r}^{h}\left(\left|y_{r, d}^{\tau-l}\right|>\zeta, \tilde{P}_{2}^{\tau-l}=0\right)}{P_{r}^{h}\left(\left|y_{r, d}^{\tau-l}\right|>\zeta\right)} .
\end{array}
$$

Substituting (13) and (37) into (44), we have

$$
P_{r}^{h}\left(\Phi_{6}\right)=\frac{\exp \left(-2 \zeta^{2} / \mathcal{N}_{0}\right) \Psi\left(\gamma_{s, r}^{\tau}\right)}{\Gamma\left(P_{1}\left|h_{s, r}^{\tau}\right|^{2}, P_{2}\left|h_{r, d}^{\tau}\right|^{2}\right)} .
$$

\section{Average BER Formulation}

We provide in this section the conditional BER given the occurrence of each scenario $\Phi_{i}$ for $i=1,2, \ldots, 6$. Using the obtained conditional BER and the probability of occurrence of each scenario from the previous section, we then derive the average BER formulation for the proposed differential DF scheme. The analysis is based on the assumption of perfect judgment on each decoded symbol at the relay; the obtained average BER will serve as the performance benchmark that the proposed scheme is able to achieve when the error propagation is negligible at the relay.

1) Conditional BER of Each Scenario: When the scenario $\Phi_{1}$ occurs, the destination estimates the transmitted symbol $v_{m}$ by using only the received signal $y_{s, d}^{\tau}$ from the source. Let $\gamma_{i}$ represents the instantaneous SNR given that the scenario $\Phi_{i}$ occurs. Based on the conditional BER formulation for DMPSK signals with single-channel reception [18], the conditional BER for the scenario $\Phi_{1}$ is

$$
\left.P_{\mathrm{BER}}^{h}\right|_{\Phi_{1}}=\Omega_{1}\left(\gamma_{1}\right) \triangleq \frac{1}{4 \pi} \int_{-\pi}^{\pi} f_{1}(\theta) \exp \left[-\alpha(\theta) \gamma_{1}\right] d \theta
$$

where the $\operatorname{SNR} \gamma_{1}$ is specified as [18]

$$
\begin{aligned}
\gamma_{1} & =\frac{P_{1}\left|h_{s, d}^{\tau}\right|^{2}}{\mathcal{N}_{0}} \\
f_{1}(\theta) & =\frac{1-\beta^{2}}{1+2 \beta \sin \theta+\beta^{2}}
\end{aligned}
$$

and

$$
\alpha(\theta)=\frac{b^{2}}{2 \log _{2} M}\left(1+2 \beta \sin \theta+\beta^{2}\right)
$$

in which $M$ is the constellation size. In (48) and (49), the parameter $\beta=a / b$ is a constant whose value depends on constellation size. For example, $a=10^{-3}$ and $b=\sqrt{2}$ for DBPSK modulation, and $a=\sqrt{2-\sqrt{2}}$ and $b=\sqrt{2+\sqrt{2}}$ for DQPSK modulation [18]. The values of $a$ and $b$ for larger constellation sizes can be obtained by using the result in [1].

The scenarios $\Phi_{2}$ to $\Phi_{6}$ correspond to the case that the destination combines the received signal $y_{s, d}^{\tau}$ from the source and $y_{r, d}^{\tau}$ from the relay. The conditional BER for these scenarios depends on the combining weights $a_{1}$ and $a_{2}$ [see (7)]. Under the scenario $\Phi_{2}$, the received signals from the source and the relay can be expressed as

$$
\begin{aligned}
& y_{s, d}^{\tau}=v_{m} y_{s, d}^{\tau-1}+\tilde{w}_{s, d}^{\tau} \\
& y_{r, d}^{\tau}=v_{m} y_{r, d}^{\tau-l}+\tilde{w}_{r, d}^{\tau}
\end{aligned}
$$

where $\tilde{w}_{s, d}^{\tau}$ and $\tilde{w}_{r, d}^{\tau}$ are the additive noise terms, and each of them is zero-mean Gaussian distributed with variance $2 \mathcal{N}_{0}$. Since the noise terms of the received signals from both direct link and relay link have the same mean and variance, the SNR

$$
P_{r}^{h}\left(\Phi_{4}\right)=\frac{\mathcal{M}\left(P_{2}\left|h_{r, d}^{\tau}\right|^{2}, \zeta\right) e^{-\zeta^{2} / \mathcal{N}_{0}} \Psi\left(\gamma_{s, r}^{\tau}\right)\left(1-\Psi\left(\gamma_{s, r}^{\tau}\right)\right)}{\Gamma\left(P_{1}\left|h_{s, r}^{\tau}\right|^{2}, P_{2}\left|h_{r, d}^{\tau}\right|^{2}\right)}
$$

$$
\begin{aligned}
P_{r}^{h}\left(\Phi_{5}\right) & =P_{r}^{h}\left(\left|y_{r, d}^{\tau}\right|>\zeta, \tilde{P}_{2}^{\tau}=0, \tilde{P}_{2}^{\tau-l}=P_{2},|| y_{r, d}^{\tau-l} \mid>\zeta\right) \\
& =\frac{P_{r}^{h}\left(\left|y_{r, d}^{\tau}\right|>\zeta, \tilde{P}_{2}^{\tau}=0\right) P_{r}^{h}\left(\left|y_{r, d}^{\tau-l}\right|>\zeta, \tilde{P}_{2}^{\tau-l}=P_{2}\right)}{P_{r}^{h}\left(\left|y_{r, d}^{\tau-l}\right|>\zeta\right)}
\end{aligned}
$$


of the combiner output under the scenario $\Phi_{2}$ can be maximized by choosing the combining weights $a_{1}=a_{2}=\left(1 / 2 \mathcal{N}_{0}\right)$. These combining weights are not optimum in general. However, they are optimum in a scenario when signals from both direct and relay links contains information and their corresponding channel variances have the same values, i.e., $\sigma_{s, d}^{2}=\sigma_{r, d}^{2}$. With combining weights $a_{1}=a_{2}=\left(1 / 2 \mathcal{N}_{0}\right)$, the conditional BER can be determined by using the conditional BER formulation for DMPSK signals with two-channel reception [18]. Thus, we can express $\left.P_{\mathrm{BER}}^{h}\right|_{\Phi_{2}}$ as

$$
\left.P_{\mathrm{BER}}^{h}\right|_{\Phi_{2}}=\Omega_{2}\left(\gamma_{2}\right) \triangleq \frac{1}{16 \pi} \int_{-\pi}^{\pi} f_{2}(\theta) \exp \left[-\alpha(\theta) \gamma_{2}\right] d \theta
$$

where

$$
f_{2}(\theta)=\frac{b^{2}\left(1-\beta^{2}\right)\left[3+\cos (2 \theta)-\left(\beta+\frac{1}{\beta}\right) \sin (\theta)\right]}{2 \alpha(\theta)}
$$

and $\alpha(\theta)$ is specified in (49). The instantaneous $\mathrm{SNR} \gamma_{2}$ is given by

$$
\gamma_{2}=\frac{P_{1}\left|h_{s, d}^{\tau}\right|^{2}}{\mathcal{N}_{0}}+\frac{P_{2}\left|h_{r, d}^{\tau}\right|^{2}}{\mathcal{N}_{0}}
$$

For the remaining scenarios, namely $\Phi_{3}$ to $\Phi_{6}$, the destination also combines the received signal from the source and that from the relay. However, the use of the two-channel differential detection for these four cases are not guaranteed to be optimum since either the received signals $y_{r, d}^{\tau}$ or $y_{r, d}^{\tau-l}$ from the relay contain only noise. Up to now, the conditional BER formulation for DMPSK with arbitrary-weighted combining has not been available in the literature. For analytical tractability of the analysis, we resort to an approximate BER, in which the signal from the relay is considered as noise when $\Phi_{3}$ to $\Phi_{6}$ occur. As we will show in the succeeding section, the analytical BER obtained from this approximation is very close to the simulation results. The approximate conditional BER for the scenarios $\Phi_{\nu}$, $\nu=3, \ldots, 6$, are $\left.P_{\mathrm{BER}}^{h}\right|_{\Phi_{\nu}} \approx \Omega_{2}\left(\gamma_{\nu}\right)$, where

$$
\gamma_{\nu}=\frac{P_{1}\left|h_{s, d}^{\tau}\right|^{2}}{\mathcal{N}_{0}+\frac{\mathcal{N}_{\nu}}{P_{1}\left|h_{s, d}^{\tau}\right|^{2} / \mathcal{N}_{0}}}
$$

in which $\mathcal{N}_{\nu}$ represents the noise power that comes from the relay link given that the scenario $\Phi_{\nu}$ occurs. The noise power $\mathcal{N}_{\nu}$ depends on the received signal from the relay at the current time $\tau\left(y_{r, d}^{\tau}\right)$ and that stored in the memory $\left(y_{r, d}^{\tau-l}\right)$. Under the scenario $\Phi_{3}, \mathcal{N}_{3}$ is given by $\left(P_{2}\left|h_{r, d}\right|^{2}+\mathcal{N}_{0}\right)^{2} / \mathcal{N}_{0}$. Under the scenarios $\Phi_{4}$ and $\Phi_{5}$, the relay does not send the information at either time $\tau$ or time $\tau-l$. These two scenarios result in the same noise power such that $\mathcal{N}_{4}=\mathcal{N}_{5}=P_{2}\left|h_{r, d}\right|^{2}+\mathcal{N}_{0}$. The last scenario, i.e., $\Phi_{6}$, corresponds to the case when both $y_{r, d}^{\tau}$ and $y_{r, d}^{\tau-l}$ does not contain any information symbol. The noise power $\mathcal{N}_{6}$ is equal to the noise variance $\mathcal{N}_{0}$.

2) Average BER: In what follows, we determine the average BER $P_{\mathrm{BER}}^{(i)} \triangleq \mathrm{E}\left[P_{\mathrm{BER}}^{h} \mid \Phi_{i} P_{r}^{h}\left(\Phi_{i}\right)\right]$ for each scenario. Assuming that the fading channels of different transmit-receive links are independent, the average BER for the scenario $\Phi_{1}$ can be obtained by using (22) and (46) such that

$$
\begin{aligned}
P_{\mathrm{BER}}^{(1)}= & \mathrm{E}\left[\Omega_{1}\left(\frac{P_{1}\left|h_{s, d}^{\tau}\right|^{2}}{\mathcal{N}_{0}}\right)\right] \\
& \times \mathrm{E}\left[\left(1-e^{-\zeta^{2} / \mathcal{N}_{0}}\right) \Psi\left(\frac{P_{1}\left|h_{s, r}^{\tau}\right|^{2}}{\mathcal{N}_{0}}\right)\right. \\
& +\left(1-\mathcal{M}\left(P_{2}\left|h_{r, d}^{\tau}\right|^{2}, \zeta\right)\right) \\
& \left.\times\left(1-\Psi\left(\frac{P_{1}\left|h_{s, r}^{\tau}\right|^{2}}{\mathcal{N}_{0}}\right)\right)\right] .
\end{aligned}
$$

Averaging over the Rayleigh fading channels $h_{s, d}^{\tau}, h_{s, r}^{\tau}$, and $h_{r, d}^{\tau}$, the average BER under the scenario $\Phi_{1}$ is given by

$$
\begin{aligned}
P_{\mathrm{BER}}^{(1)}= & F_{1}\left(1+\frac{\alpha(\theta) P_{1} \sigma_{s, d}^{2}}{\mathcal{N}_{0}}\right) \\
& \times\left[\left(1-e^{\frac{-\zeta^{2}}{\mathcal{N}_{0}}}\right) G\left(1+g(\theta) P_{1} \sigma_{s, r}^{2} / \mathcal{N}_{0}\right)\right. \\
& +\left(1-\int_{0}^{\infty} \frac{\mathcal{M}\left(P_{2} q, \zeta\right)}{\sigma_{r, d}^{2}} e^{\frac{-q}{\sigma_{r, d}^{2}}} d q\right) \\
& \left.\times\left(1-G\left(1+\frac{g(\theta) P_{1} \sigma_{s, r}^{2}}{\mathcal{N}_{0}}\right)\right)\right]
\end{aligned}
$$

in which $F_{1}(c(\theta))=(1 / 4 \pi) \int_{-\pi}^{\pi} f_{1}(\theta) / c(\theta) d \theta$, and $G(c(\theta))=$ $(1 / \pi) \int_{0}^{(M-1) \pi / M} c(\theta)^{-1} d \theta$, in which $c(\theta)$ is a function of $\theta$.

According to (31) and (52), the average BER under the scenario $\Phi_{2}$ can be approximated as

$$
\begin{aligned}
P_{\mathrm{BER}}^{(2)} & \approx \mathrm{E}\left[\Omega_{2}\left(\frac{P_{1}\left|h_{s, d}^{\tau}\right|^{2}}{\mathcal{N}_{0}}+\frac{P_{2}\left|h_{r, d}^{\tau}\right|^{2}}{\mathcal{N}_{0}}\right)\right. \\
& \left.\times \frac{\mathcal{M}^{2}\left(P_{2}\left|h_{r, d}^{\tau}\right|^{2}, \zeta\right)\left(1-\Psi\left(\frac{P_{1}\left|h_{s, r}\right|^{2}}{\mathcal{N}_{0}}\right)\right)^{2}}{1-\left(1-e^{-\zeta^{2} / \mathcal{N}_{0}}\right) \Psi\left(\frac{P_{1}\left|h_{s, r}\right|^{2}}{\mathcal{N}_{0}}\right)}\right] .
\end{aligned}
$$

By averaging over all channel coefficients, we have

$$
\begin{aligned}
P_{\mathrm{BER}}^{(2)} & \approx \frac{1}{\sigma_{r, d}^{2}} \int_{0}^{\infty} s_{2}(z) \mathcal{M}^{2}\left(P_{2} z, \zeta\right) e^{-\frac{z}{\sigma_{r, d}^{2}}} d z \\
& \times \frac{1}{\sigma_{s, r}^{2}} \int_{0}^{\infty} \frac{\left(1-\Psi\left(\frac{P_{1} y}{\mathcal{N}_{0}}\right)\right)^{2}}{1-\left(1-e^{-\zeta^{2} / \mathcal{N}_{0}}\right) \Psi\left(\frac{P_{1} y}{\mathcal{N}_{0}}\right)} \\
& \times e^{-\frac{y}{\sigma_{s, r}^{2}}} d y
\end{aligned}
$$

where

$$
s_{2}(q)=\frac{1}{16 \pi} \int_{-\pi}^{\pi} \frac{f_{2}(\theta)}{1+\frac{\alpha(\theta) P_{1} \sigma_{s, d}^{2}}{\mathcal{N}_{0}}} e^{-\alpha(\theta) \frac{P_{2} q}{\mathcal{N}_{0}}} d \theta .
$$


The average BER under the scenario $\Phi_{3}$ can be approximated by $P_{\mathrm{BER}}^{(3)} \approx \mathrm{E}\left[\Omega_{2}\left(\gamma_{3}\right)\left(P_{r}^{h}\left(\Phi_{2} \cup \Phi_{3}\right)-P_{r}^{h}\left(\Phi_{2}\right)\right)\right]$. From (31), (39), and (55), we can approximate the average BER under the scenario $\Phi_{3}$ as

$$
\begin{aligned}
P_{\mathrm{BER}}^{(3)} \approx & \mathrm{E}\left[\Omega_{2}\left(\frac{P_{1}\left|h_{s, d}^{\tau}\right|^{2}}{\mathcal{N}_{0}+\frac{\left(P_{2}\left|h_{r, d}^{\tau}\right|^{2}+\mathcal{N}_{0}\right)^{2}}{P_{1}\left|h_{s, d}^{\tau}\right|^{2}}}\right)\right. \\
& \times \mathcal{M}^{2}\left(P_{2}\left|h_{r, d}^{\tau}\right|^{2}, \zeta\right) \\
& \times\left(1-\Psi\left(\frac{P_{1}\left|h_{s, r}^{\tau}\right|^{2}}{\mathcal{N}_{0}}\right)\right)^{2} \\
& \times\left(\frac{1}{\Gamma\left(P_{1}\left|h_{s, r}^{\tau}\right|^{2}, P_{2}\left|h_{r, d}^{\tau}\right|^{2}\right)}\right. \\
& \left.\left.-\frac{1}{1-\left(1-e^{-\zeta^{2} / \mathcal{N}_{0}}\right) \Psi\left(\frac{P_{1}\left|h_{s, r}^{\tau}\right|^{2}}{\mathcal{N}_{0}}\right)}\right)\right] .
\end{aligned}
$$

Average (61) over all channel coefficients, resulting in

$$
P_{\mathrm{BER}}^{(3)} \approx \frac{1}{\sigma_{s, r}^{2}} \int_{0}^{\infty}\left[\frac{1}{16 \pi} \int_{-\pi}^{\pi} f_{2}(\theta) s_{3}(u, \theta) d \theta\right] e^{-u / \sigma_{s, r}^{2}} d u
$$

where

$$
\begin{aligned}
s_{3}(y, \theta) & \frac{1}{\sigma_{s, d}^{2} \sigma_{r, d}^{2}} \int_{0}^{\infty} \int_{0}^{\infty} \exp \left(-\frac{\alpha(\theta) P_{1} x}{\mathcal{N}_{0}+\frac{\left(P_{2} z+\mathcal{N}_{0}\right)^{2}}{P_{1} x}}\right. \\
& \left.-\frac{x}{\sigma_{s, d}^{2}}-\frac{z}{\sigma_{r, d}^{2}}\right) \\
& \times \mathcal{M}^{2}\left(P_{2} z, \zeta\right)\left(1-\Psi\left(\frac{P_{1} y}{\mathcal{N}_{0}}\right)\right)^{2} \\
& \times\left(\frac{1}{\Gamma\left(P_{1} y, P_{2} z\right)}\right. \\
& \left.-\frac{1}{1-\left(1-e^{-\zeta^{2} / \mathcal{N}_{0}}\right) \Psi\left(\frac{P_{1} y}{\mathcal{N}_{0}}\right)}\right) d x d z .
\end{aligned}
$$

Under the scenario $\Phi_{4}$, the approximate BER can be expressed as

$$
\begin{aligned}
P_{\mathrm{BER}}^{(4)} \approx & \mathrm{E}\left[\Omega_{2}\left(\frac{P_{1}\left|h_{s, d}\right|^{2}}{\mathcal{N}_{0}+\frac{P_{2}\left|h_{r, d}\right|^{2}+\mathcal{N}_{0}}{P_{1}\left|h_{s, d}\right|^{2} / \mathcal{N}_{0}}}\right)\right. \\
& \times e^{-\zeta^{2} / \mathcal{N}_{0}} \mathcal{M}\left(P_{2}\left|h_{r, d}^{\tau}\right|^{2}, \zeta\right) \\
& \left.\times \frac{\Psi\left(\frac{P_{1}\left|h_{s, r}\right|^{2}}{\mathcal{N}_{0}}\right)\left(1-\Psi\left(\frac{P_{1}\left|h_{s, r}\right|^{2}}{\mathcal{N}_{0}}\right)\right)}{\Gamma\left(P_{1}\left|h_{s, r}^{\tau}\right|^{2}, P_{2}\left|h_{r, d}^{\tau}\right|^{2}\right)}\right] .
\end{aligned}
$$

By averaging over all channel coefficients, we obtain

$$
P_{\mathrm{BER}}^{(4)} \approx \frac{1}{\sigma_{s, r}^{2}} \int_{0}^{\infty}\left[\frac{1}{16 \pi} \int_{-\pi}^{\pi} f_{2}(\theta) s_{4}(u, \theta) d \theta\right] e^{-u / \sigma_{s, r}^{2}} d u
$$

where

$$
\begin{aligned}
s_{4}(u, \theta) & \\
= & \frac{1}{\sigma_{s, d}^{2} \sigma_{r, d}^{2}} \int_{0}^{\infty} \int_{0}^{\infty} \exp \left(-\frac{\alpha(\theta) P_{1} z}{\mathcal{N}_{0}+\frac{P_{2} q+\mathcal{N}_{0}}{P_{1} z / \mathcal{N}_{0}}}\right. \\
& \left.-\frac{\zeta^{2}}{\mathcal{N}_{0}}-\frac{z}{\sigma_{s, d}^{2}}-\frac{q}{\sigma_{r, d}^{2}}\right) \mathcal{M}\left(P_{2} q, \zeta\right) \\
& \times \frac{\Psi\left(P_{1} u / \mathcal{N}_{0}\right)\left(1-\Psi\left(P_{1} u / \mathcal{N}_{0}\right)\right)}{\Gamma\left(P_{1} u, P_{2} q\right)} d q d z .
\end{aligned}
$$

Based on the chance that the scenario $\Phi_{5}$ occurs and the conditional bit error probability $\left.P_{\mathrm{BER}}^{h}\right|_{\Phi_{5}}$, we can see that the approximate BER under the scenario $\Phi_{5}$ is the same as that under the scenario $\Phi_{4}$, and, hence, $P_{\mathrm{BER}}^{(5)}$ can be expressed as in (64). From (45) and (55), the average BER under the scenario $\Phi_{6}$ can be approximate as

$$
\begin{array}{r}
P_{\mathrm{BER}}^{(6)} \approx \mathrm{E}\left[\Omega_{2}\left(\frac{P_{1}\left|h_{s, d}^{\tau}\right|^{2}}{\mathcal{N}_{0}+\frac{\mathcal{N}_{0}}{P_{1}\left|h_{s, d}^{\tau}\right|^{2} / \mathcal{N}_{0}}}\right)\right. \\
\\
\left.\times \frac{e^{-2 \zeta^{2} / \mathcal{N}_{0}} \Psi\left(\frac{P_{1}\left|h_{s, r}^{\tau}\right|^{2}}{\mathcal{N}_{0}}\right)^{2}}{\Gamma\left(P_{1}\left|h_{s, r}^{\tau}\right|^{2}, P_{2}\left|h_{r, d}^{\tau}\right|^{2}\right)}\right] .
\end{array}
$$

By averaging over all channel coefficients, we have

$$
\begin{aligned}
P_{\mathrm{BER}}^{(6)} & \approx\left[\frac{1}{16 \pi} \int_{-\pi}^{\pi} f_{2}(\theta) s_{6}(\theta) d \theta\right] e^{-2 \zeta^{2} / \mathcal{N}_{0}} \\
& \times \frac{1}{\sigma_{s, r}^{2}} \frac{1}{\sigma_{r, d}^{2}} \int_{0}^{\infty} \int_{0}^{\infty} \frac{\Psi^{2}\left(\frac{P_{1} y}{\mathcal{N}_{0}}\right)}{\Gamma\left(P_{1} y, P_{2} z\right)} \\
& \times e^{-\frac{y}{\sigma_{s, r}^{2}}-\frac{z}{\sigma_{r, d}^{2}}} d y d z
\end{aligned}
$$

where

$$
s_{6}(\theta)=\frac{1}{\sigma_{s, d}^{2}} \int_{0}^{\infty} \exp \left(-\frac{\alpha(\theta) P_{1} z}{\mathcal{N}_{0}+\frac{\mathcal{N}_{0}}{P_{1} z / \mathcal{N}_{0}}}-\frac{z}{\sigma_{s, d}^{2}}\right) d z .
$$

To this end, we have completed the derivation of the average $\mathrm{BER}$ for all possible scenarios. Finally, the average BER, $P_{\mathrm{BER}}$, can be determined by summing together the average BER $P_{\mathrm{BER}}^{(i)}$ for $i=1,2, \ldots, 6$

$$
P_{\mathrm{BER}}=P_{\mathrm{BER}}^{(1)}+P_{\mathrm{BER}}^{(2)}+P_{\mathrm{BER}}^{(3)}+2 P_{\mathrm{BER}}^{(4)}+P_{\mathrm{BER}}^{(6)}
$$

in which $P_{\mathrm{BER}}^{(i)}$ are specified in (57), (59), (62), (65), and (68). The average BER in (70) is based on an assumption of perfect judgment on each decoded symbol at the relay. As will be shown later, the BER in (70) is the best BER performance that the proposed scheme is able to achieve.

\section{BER UPPER BOUND AND BER LOWER BOUND}

We provide in this section BER upper bound and BER lower bound of the proposed threshold-based differential DF scheme. 
Then, we show through computer simulation that, when the power allocation and the decision threshold are properly designed, the BER upper bound and the BER lower bound are close to the simulated BER.

To obtain a BER upper bound, we first note that the conditional BER for each scenario, $\left.P_{\mathrm{BER}}^{h}\right|_{\Phi_{i}}$, is at most $1 / 2$. In addition, if the threshold is properly designed, then the chances that the scenarios $\Phi_{3}$ to $\Phi_{6}$ happen are small compared to the chances that the scenarios $\Phi_{1}$ and $\Phi_{2}$ happen. Therefore, the BER upper bound can be obtained by bounding the conditional BER $\left.P_{\mathrm{BER}}^{h}\right|_{\Phi_{3}},\left.P_{\mathrm{BER}}^{h}\right|_{\Phi_{4}},\left.P_{\mathrm{BER}}^{h}\right|_{\Phi_{5}}$, and $\left.P_{\mathrm{BER}}^{h}\right|_{\Phi_{6}}$ by $1 / 2$, we have

$$
P_{\mathrm{BER}} \leq P_{\mathrm{BER}}^{(1)}+P_{\mathrm{BER}}^{(2)}+\frac{1}{2} \sum_{\nu=3}^{6} P_{r}^{h}\left(\Phi_{\nu}\right)
$$

where $P_{\mathrm{BER}}^{(1)}$ and $P_{\mathrm{BER}}^{(2)}$ are determined in (57) and (59), and $P_{r}^{h}\left(\Phi_{\nu}\right), \nu=3,4,5$, and 6 , are given in (32), (42), (43), and (45), respectively.

To find a BER lower bound, we found that it is hard to obtain the exact BER of $P_{\mathrm{BER}}^{(2)}$ and $P_{\mathrm{BER}}^{(3)}$ because the exact expressions of the chances that the scenarios $\Phi_{2}$ and $\Phi_{3}$ occur involve the approximation that the channel coefficients are constant for several symbol periods. In addition, the BER formulation given that the scenario $\Phi_{3}$ happens is currently unavailable. However, if the power ratio and the threshold are properly designed, the chance that the scenario $\Phi_{3}$ occurs tends to be small compared to the chance that the scenario $\Phi_{2}$ occurs. Moreover, the conditional BER under the scenario $\Phi_{3}\left(\left.P_{\mathrm{BER}}^{h}\right|_{\Phi_{3}}\right)$ is larger than that under the scenario $\Phi_{2}\left(P_{\mathrm{BER}}^{h} \mid \Phi_{2}\right)$. Therefore, the BER lower bound can be obtained by bounding the conditional BER $P_{\mathrm{BER}}^{h} \mid \Phi_{3}$ with $\left.P_{\mathrm{BER}}^{h}\right|_{\Phi_{2}}$. In this way, the BER under the scenarios $\Phi_{2}$ and $\Phi_{3}$ can be lower bounded as

$$
P_{\mathrm{BER}}^{(2)}+P_{\mathrm{BER}}^{(3)} \geq \mathrm{E}\left[\left.P_{\mathrm{BER}}^{h}\right|_{\Phi_{2}} P_{r}^{h}\left(\Phi_{2} \cup \Phi_{3}\right)\right]
$$

where $P_{r}^{h}\left(\Phi_{2} \cup \Phi_{3}\right)$, which is evaluated in (39), is the chance that the scenarios $\Phi_{2}$ and $\Phi_{3}$ occur. According to (31) and (52), the average BER in (72) can be expressed as

$$
\begin{aligned}
P_{\mathrm{BER}}^{(2)}+P_{\mathrm{BER}}^{(3)} \geq \mathrm{E}\left[\Omega_{2}\left(\frac{P_{1}\left|h_{s, d}^{\tau}\right|^{2}}{\mathcal{N}_{0}}+\frac{P_{2}\left|h_{r, d}^{\tau}\right|^{2}}{\mathcal{N}_{0}}\right)\right. \\
\left.\times \frac{\mathcal{M}^{2}\left(P_{2}\left|h_{r, d}^{\tau}\right|^{2}, \zeta\right)\left(1-\Psi\left(\frac{P_{1}\left|h_{s, r}^{\tau}\right|^{2}}{\mathcal{N}_{0}}\right)\right)^{2}}{\Gamma\left(P_{1}\left|h_{s, r}^{\tau}\right|^{2}, P_{2}\left|h_{r, d}^{\tau}\right|^{2}\right)}\right] .
\end{aligned}
$$

By averaging over the channel realization, we obtain

$$
\begin{aligned}
P_{\mathrm{BER}}^{(2)} & +P_{\mathrm{BER}}^{(3)} \\
\geq & \frac{1}{\sigma_{s, r}^{2}} \int_{0}^{\infty}\left[\frac{1}{16 \pi} \int_{-\pi}^{\pi} \frac{f_{2}(\theta) s(u, \theta)}{1+\alpha(\theta) \frac{P_{1} \sigma_{s, d}^{2}}{\mathcal{N}_{0}}} d \theta\right] \\
& \times e^{-\frac{u}{\sigma_{s, r}^{u}}} d u \\
\triangleq & \mathrm{LB}\left\{P_{\mathrm{BER}}^{(2)}+P_{\mathrm{BER}}^{(3)}\right\}
\end{aligned}
$$

where

$$
\begin{aligned}
s(u, \theta) & =\int_{0}^{\infty} \frac{\mathcal{M}^{2}\left(P_{2} q, \zeta\right)\left(1-\Psi\left(\frac{P_{1} u}{\mathcal{N}_{0}}\right)\right)^{2}}{\sigma_{r, d}^{2} \Gamma\left(P_{1} u, P_{2} q\right)} \\
& \times e^{-\alpha(\theta) \frac{P_{2} q}{\mathcal{N}_{0}}-\frac{q}{\sigma_{r, d}^{2}}} d q .
\end{aligned}
$$

Since the exact BER formulations under the scenarios 4,5 , and 6 are currently unavailable, and the chances that these three scenarios happen are small at high SNR, we further lower bound the BER $P_{\mathrm{BER}}^{(4)}, P_{\mathrm{BER}}^{(5)}$, and $P_{\mathrm{BER}}^{(6)}$ by 0 . As a result, the BER of the proposed differential DF scheme can be lower bounded by

$$
P_{\mathrm{BER}} \geq P_{\mathrm{BER}}^{(1)}+\mathrm{LB}\left\{P_{\mathrm{BER}}^{(2)}+P_{\mathrm{BER}}^{(3)}\right\}
$$

where $P_{\mathrm{BER}}^{(1)}$ is determined in (57).

Fig. 3(a)-(f) compares the BER approximation (70), the BER upper bound (71), and the BER lower bound (76) with the simulated performance in the case of DQPSK modulation. In Fig. 3(a)-(c), we consider the differential DF cooperation system with $\sigma_{s, d}^{2}=\sigma_{s, r}^{2}=\sigma_{r, d}^{2}=1$, i.e., all the channel links have the same qualities. We can see from the figures that the approximate BER closely matches with the simulated BER, and both the approximate BER and the simulated BER lie between the BER upper bound and the BER lower bound. Moreover, the system performance depends on the power allocation and the threshold. By choosing proper power allocation and threshold, not only the BER performance improves, but also the lower bound is closer to the simulated performance. For example, considering a system with threshold of $\zeta=1$ and total transmit power $P_{1}+P_{2}=P$ where $P_{1}$ and $P_{2}$ are transmitted power of the source and the relay, respectively. By changing the power allocation from $P_{1} / P=0.5$ to $P_{1} / P=0.8$, the BER performance is improved by $1 \mathrm{~dB}$ at a BER of $10^{-4}$, while the performance gap between the simulated BER and the BER lower bound is reduced by $2 \mathrm{~dB}$ at the same BER. This result follows the fact that when the threshold $\zeta$ is appropriately chosen, the scenarios $\Phi_{3}$ to $\Phi_{6}$ occur with much smaller probability than the scenarios $\Phi_{1}$ and $\Phi_{2}$. Even though the BER under each of the scenarios $\Phi_{3}$ to $\Phi_{6}$ is larger than that under the scenarios $\Phi_{1}$ or $\Phi_{2}$, the average BER $P_{\mathrm{BER}}^{(\nu)}, \nu=3, \ldots, 6$ are smaller. The BER $P_{\mathrm{BER}}$ is dominated by the BER under scenarios $\Phi_{1}$ and $\Phi_{2}$. Therefore, the performance gaps between the bounds and the approximate BER is small if the threshold is properly designed.

We have the same observations in Fig. 3(d)-(f) for a system with $\sigma_{s, d}^{2}=1, \sigma_{s, r}^{2}=1$, and $\sigma_{r, d}^{2}=10$. At a threshold of $\zeta=1$, the performance can be improved by allocating more power at the source and less power at the relay. This is in agreement with the results in [10] which illustrate that the channel link between source and relay and the channel link between relay and destination should be balanced in order to achieve a performance diversity of two. Interestingly, the performance can be significantly improved by choosing a proper threshold regardless of the power allocation. For instance, increasing the threshold from 1 to 2 , the performance of the proposed scheme with equal power allocation improves $4 \mathrm{~dB}$ at a BER of $10^{-4}$. At the threshold of $\zeta=2$, by changing the power allocation 


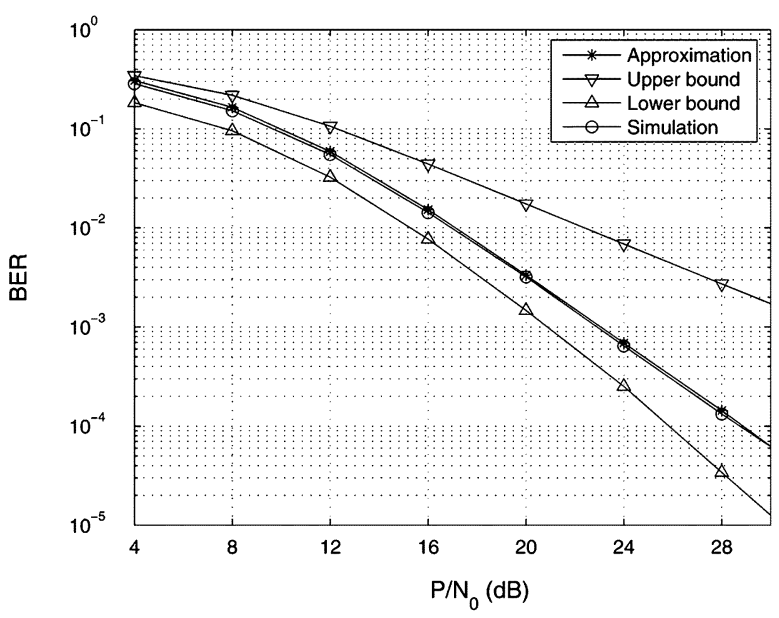

(a)

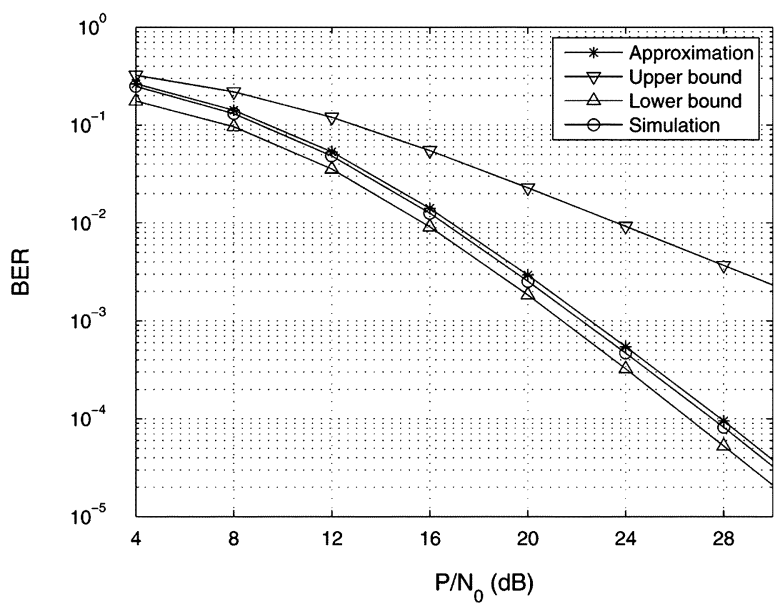

(b)

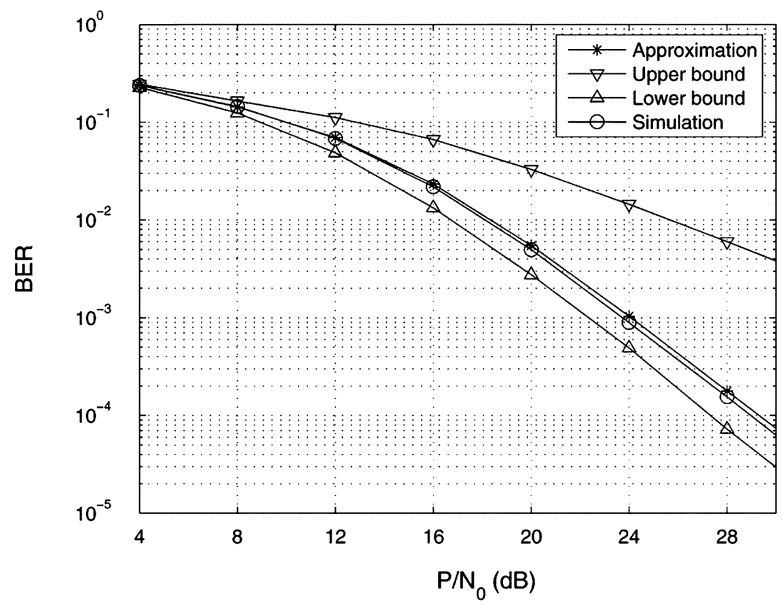

(c)

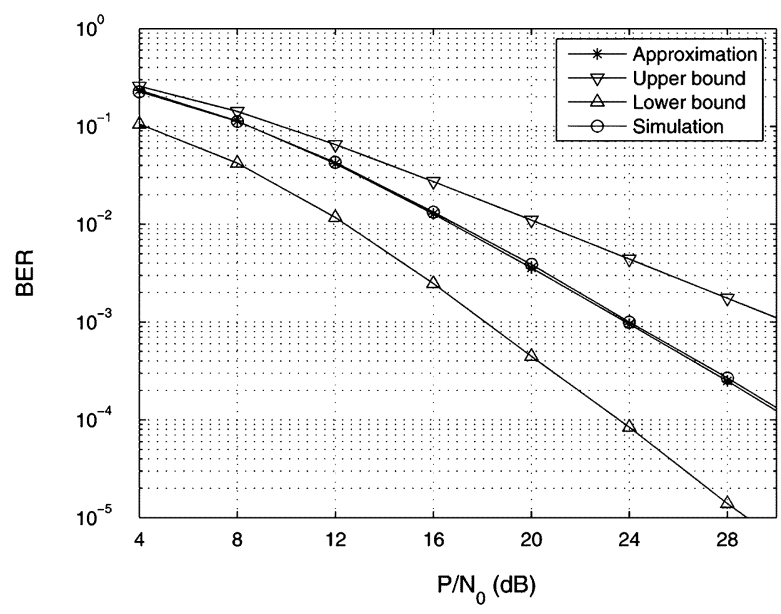

(d)

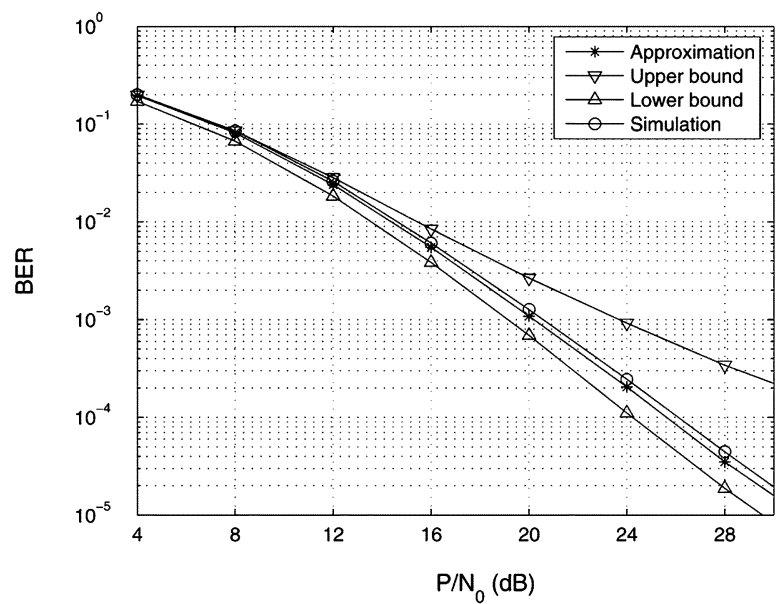

(e)

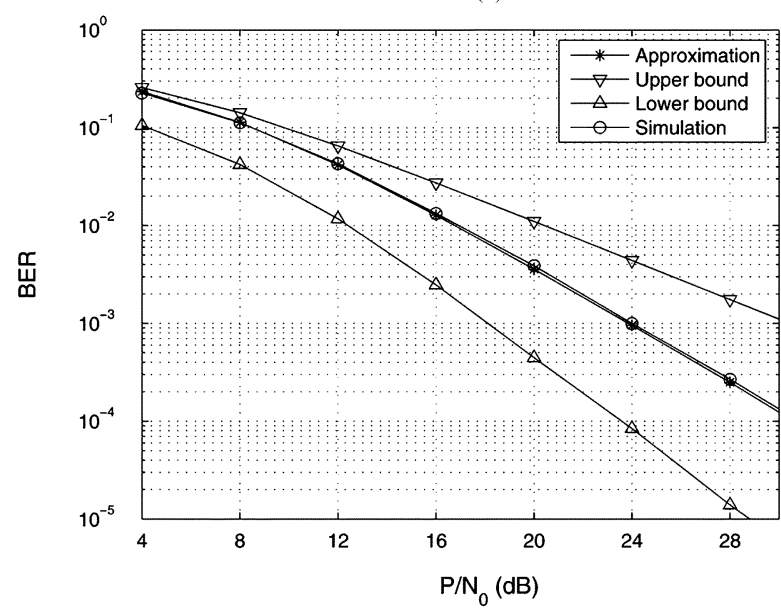

(f)

Fig. 3. DQPSK cooperation system: $\sigma_{s, d}^{2}=1, \sigma_{s, r}^{2}=1$. (a) $P_{1}=0.5 P, P_{2}=0.5 P, \zeta=1, \sigma_{r, d}^{2}=1$. (b) $P_{1}=0.8 P, P_{2}=0.2 P, \zeta=1, \sigma_{r, d}^{2}=1$. (c) $P_{1}=0.8 P, P_{2}=0.2 P, \zeta=2, \sigma_{r, d}^{2}=1$. (d) $P_{1}=0.5 P, P_{2}=0.5 P, \zeta=1, \sigma_{r, d}^{2}=10$. (e) $P_{1}=0.5 P, P_{2}=0.5 P, \zeta=2, \sigma_{r, d}^{2}=10$. (f) $P_{1}=$ $0.8 P, P_{2}=0.2 P, \zeta=2, \sigma_{r, d}^{2}=10$.

from $P_{1} / P=0.5$ to $P_{1} / P=0.8$, the system performance is further improved by only $0.5 \mathrm{~dB}$ at the same BER. This implies that the effect of the threshold dominates; the performance does not severely depend on the power allocation after the threshold is properly designed.

Another observation obtained from Fig. 3(a)-(f) is that the threshold depends on the channel link qualities. To be specific, the threshold should be increased as the link quality between the relay and the destination increases. For example, under the scenario $\sigma_{s, d}^{2}=\sigma_{s, r}^{2}=1$, the threshold $\zeta=1$ results in superior performance in the case of $\sigma_{r, d}^{2}=1$ while the threshold $\zeta=2$ leads to better performance in the case of $\sigma_{r, d}^{2}=10$. This observation can be explained as follows. When the link quality between the relay and the destination is good, i.e., the 
channel variance is high, the received signal from the relay tends to have large energy if it carries the information. As a result, by increasing the threshold from 1 to 2 , we reduce the chance that the received signal whose amplitude is larger than the threshold contains no information. Thus, with threshold of 2 , the received signals from the relay and the destination are efficiently combined and, hence, resulting in better performance.

\section{OPtimum Threshold AND POWER Allocation}

Simulation results in the previous section show that the choice of power allocation and threshold $\zeta$ significantly affects the performance. In this section, we determine an optimum decision threshold and an optimum power allocation for the proposed differential DF cooperation scheme based on the tight BER approximation in (70). To simplify the notation, let us denote $r=$ $P_{1} / P$ as the power ratio of the transmitted power at the source $\left(P_{1}\right)$ over the total power $(P)$. For a fixed total transmitted power $P_{1}+P_{2}=P$, we jointly optimize the threshold $\zeta$ and the power ratio $r$ such that the tight BER approximation in (70) is minimized. The optimization problem can be formulated as

$$
\min _{\zeta, r} P_{\mathrm{BER}}(\zeta, r)
$$

where $P_{\mathrm{BER}}(\zeta, r)$ represents the BER approximation as specified in (70) with $P_{1}=r P$ and $P_{2}=(1-r) P$. Note that the optimum power allocation and the optimum threshold are jointly determined based on channel variances, not instant channel coefficients. The destination needs to feedback the obtained optimum power allocation in a form of a few information bits through a control channel. The feedback information is updated only when the network topology or the channels change dramatically.

Fig. 4(a)-(f) shows the BER performance of the proposed scheme with DQPSK signals as a function of power allocation and threshold. In Fig. 4(a)-(c), we consider the case when the channel variances of all communication links are equal, i.e., $\sigma_{s, d}^{2}=\sigma_{s, r}^{2}=\sigma_{r, d}^{2}=1$. The BER approximation is plotted in Fig. 4(a) and its cross sections are shown in Fig. 4(b) and (c) together with the simulated BER curves. Based on the approximate BER in Fig. 4(a), the jointly optimum power allocation and decision threshold are $r=0.7$ and $\zeta=1$. Fig. 4(b) compares the cross-sectional curves of the approximate BER with the simulated BER in the case of $r=0.7$. We can see that the approximate BER is close to the simulated BER. Furthermore, the proposed scheme with any threshold less than 1.5 yields almost the same BER performance, and the performance significantly degrades as the threshold increases more than 1.5. The reason is that when the threshold increases, the chances that the scenarios $\Phi_{4}$ to $\Phi_{6}$ occur increases, and, hence, the average BER is dominated by the BER under these scenarios. Fig. 4(c) depicts the approximate and simulated BER curves as functions of power allocation in the case of the decision threshold $\zeta=1$. We can obviously see that the power ratio of $r=0.7$ results in the optimum performance.

In Fig. 4(d)-(f), we consider the case of channel variances $\sigma_{s, d}^{2}=1, \sigma_{s, r}^{2}=1, \sigma_{r, d}^{2}=10$. Fig. 4(d) depicts the BER of the proposed scheme as a function of the power allocation $r$ and the decision threshold $\zeta$. In this scenario, we can see that the jointly optimum power allocation and decision threshold are $r=0.8$ and $\zeta=1.7$. Fig. 4(e) shows cross-sectional curves of the approximate BER and the simulated BER performance under the same power allocation at $r=0.8$. We can see that the approximate BER closely matches to the simulated BER for every threshold value. According to both the simulated BER and the approximate BER, the optimum threshold for this case is about 1.7. We show in Fig. 4(f) a comparison of the approximate BER and the simulated BER with decision threshold $\zeta=1.7$ under different power allocation. Clearly, the approximate BER follows the same trend as the simulated BER, and the optimum power allocation is $r=0.8$ for the differential DF system with decision threshold $\zeta=1.7$.

Fig. 5(a)-(c) shows simulated and theoretical BER curves when the channel variances are $\sigma_{s, d}^{2}=1, \sigma_{s, r}^{2}=10, \sigma_{r, d}^{2}=1$ which correspond to the case when the relay is located near the source. From Fig. 5(a), we observe that the joint optimum power allocation and optimum threshold are $r=0.5$ and $\zeta=0.4$, respectively. Fig. 5(b) plots the BER curve as a function of threshold at the optimum power allocation. It confirms our expectation that in this case the threshold does not significantly influence the system performance. This is because when the relay is close to the source, the relay receives almost all the original information from the source and, hence, forwards most of the source information to the destination.

\section{Simulation Results}

We perform computer simulations of the two-user cooperation systems employing the DF protocol. The DQPSK modulation is used in all simulations. The channel fading coefficients are modeled according to the Jakes' model [21] with the Doppler frequency $f_{D}=75 \mathrm{~Hz}$ and normalized fading parameter $f_{D} T_{s}=0.0025$ where $T_{s}$ is the sampling period. The noise variance is $\mathcal{N}_{0}=1$. We generate $10^{6}$ channel realizations, and the BER is computed for each SNR value when at least 100 erroneous bits are detected. The BER curves are plotted as functions of $P / \mathcal{N}_{0}$, where $P$ is the total transmitted power. The power allocation for the source node and the relay node are fixed at $P_{1}+P_{2}=P$.

Fig. 6(a) compares the performance of the proposed threshold-based differential DF scheme to that of the differential DF scheme with ideal relay but without threshold and that of the differential DF scheme in which the relay always forwards the decoded symbol to the destination and no threshold is used. In Fig. 6, the ideal relay refers to the case that the relay is able to perfectly judge whether each decoded symbol is correct and forward only the correctly decoded symbols to the destination. We consider the system with power allocation $P_{1}=0.5 P, P_{2}=0.5 P$, and channel variances $\sigma_{s, d}^{2}=1, \sigma_{s, r}^{2}=1$, and $\sigma_{r, d}^{2}=10$. From Fig. 6(a), the proposed differential DF scheme outperforms the other two schemes. The reason is that a decoding error at the relay tends to result in an error at the destination. Hence, the performance of the differential DF scheme with always-forward relay and without threshold is worse than that of the proposed scheme. In particular, the performance degradation of $11 \mathrm{~dB}$ can be observed at a BER of $10^{-3}$. Adding a threshold at the destination can reduce the chance that the incorrectly decoded signal from 


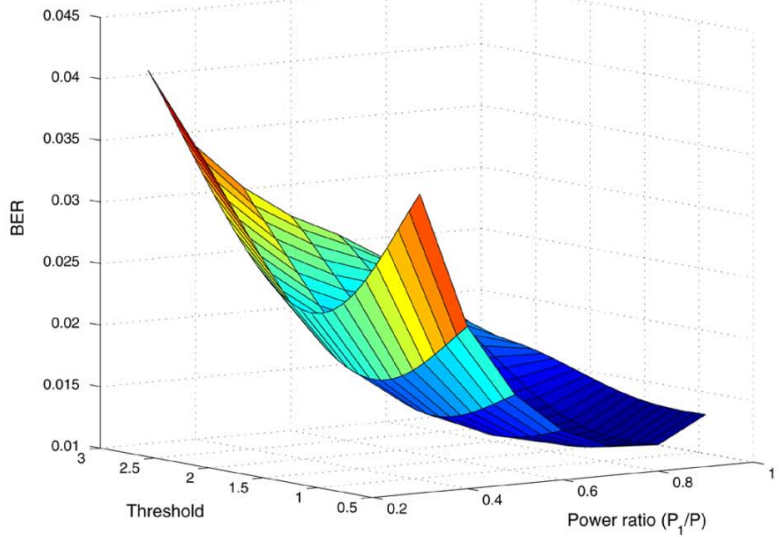

(a)

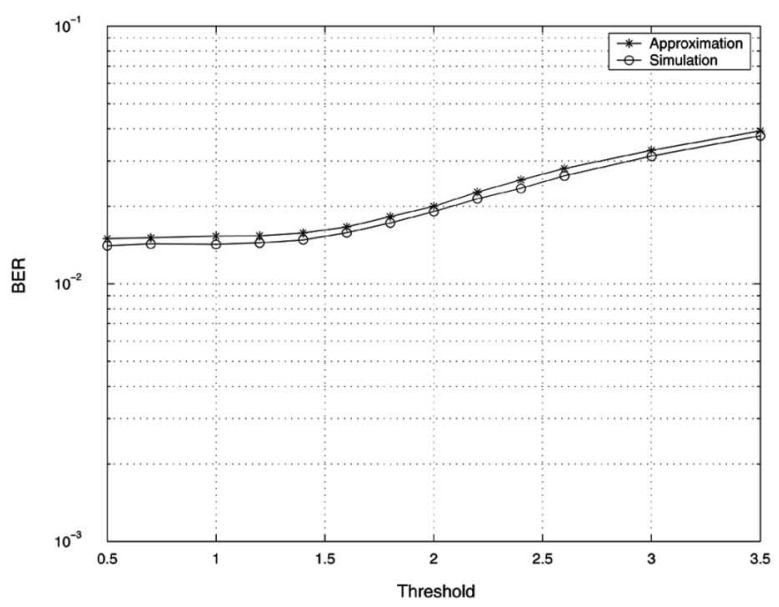

(b)

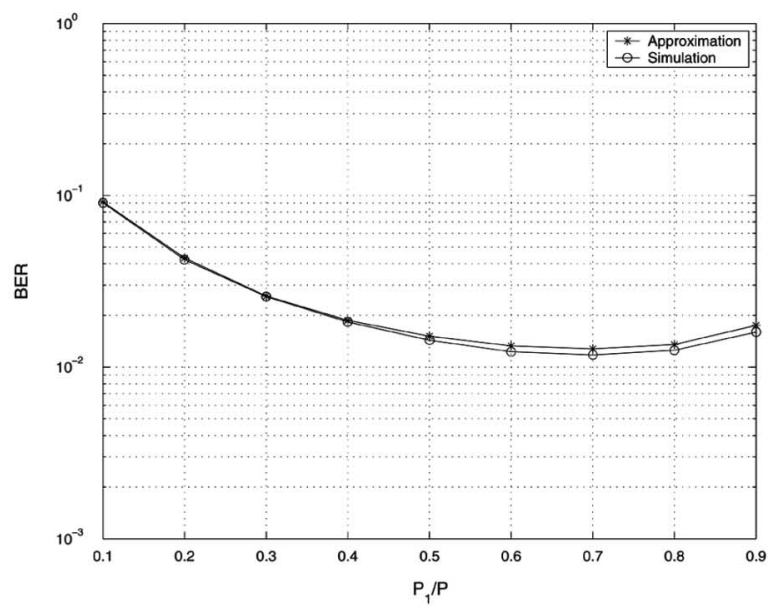

(c)

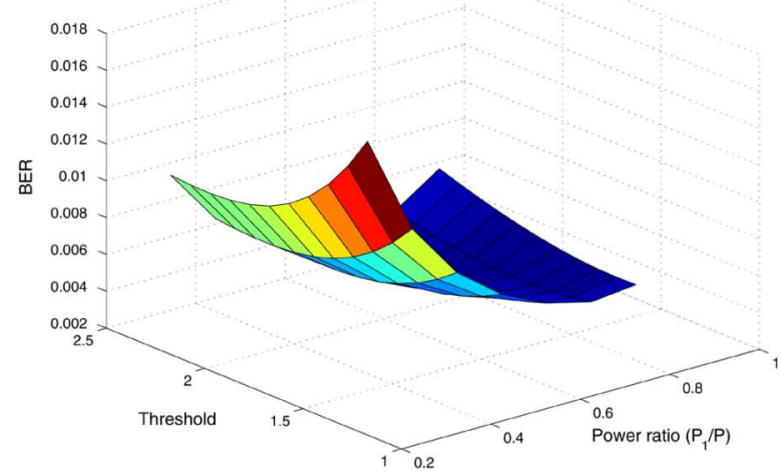

(d)

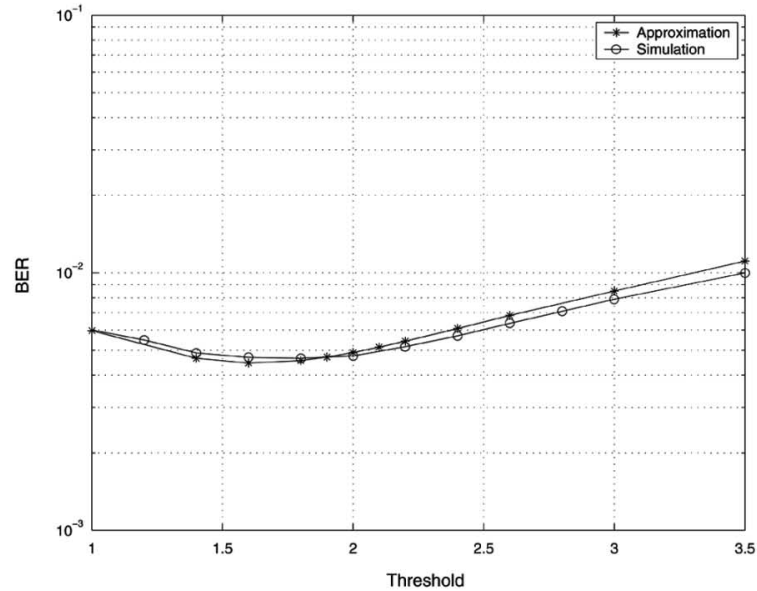

(e)

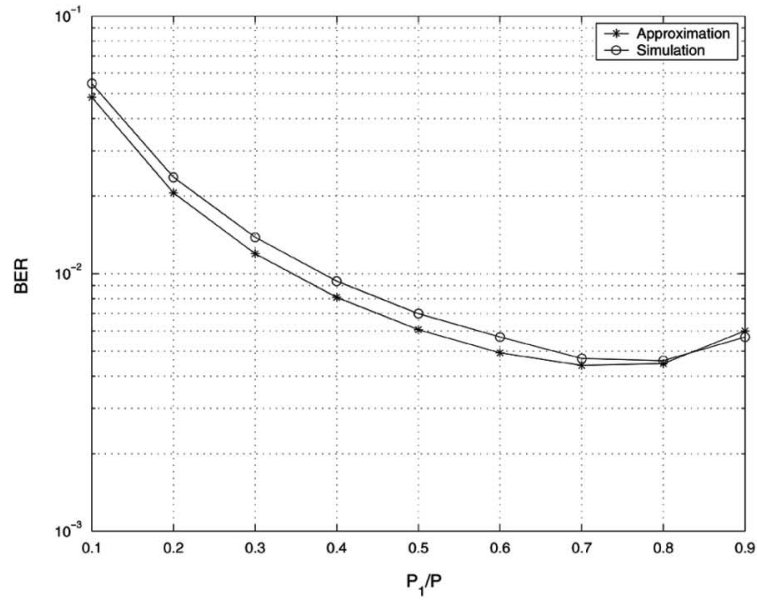

(f)

Fig. 4. DQPSK: Comparison of theoretical BER curves and simulated curves. (a)-(c) $\sigma_{s, d}^{2}=\sigma_{s, r}^{2}=\sigma_{r, d}^{2}=1$. (d)-(f) $\sigma_{s, d}^{2}=1, \sigma_{s, r}^{2}=1, \sigma_{r, d}^{2}=10$. (a) Jointly optimum threshold and power allocation. (b) Varying threshold, $P_{1}=0.7 P$ and $P_{2}=0.3 P$. (c) Varying power allocation, $\zeta=1$. (d) Jointly optimum threshold and power allocation. (e) Varying threshold, $P_{1}=0.8 P$ and $P_{2}=0.2 P$. (f) Varying power allocation, $\zeta=1.7$.

the relay is combined to the signal from the source. Therefore, the proposed scheme yields superior performance to the differential DF scheme with ideal relay but without threshold. As shown in Fig. 6(a), the proposed scheme yields about 4-dB gain at a BER of $10^{-3}$ compared to the scheme with ideal relay but without threshold. In Fig. 6(a), we also compare the performance of the proposed differential DF scheme employing DQPSK signals with that of direct transmission schemes employing differential and coherent BPSK signals. All schemes have the same bandwidth efficiency; however, the proposed differential DF scheme outperforms the direct transmission schemes with differential detection and with coherent detection 


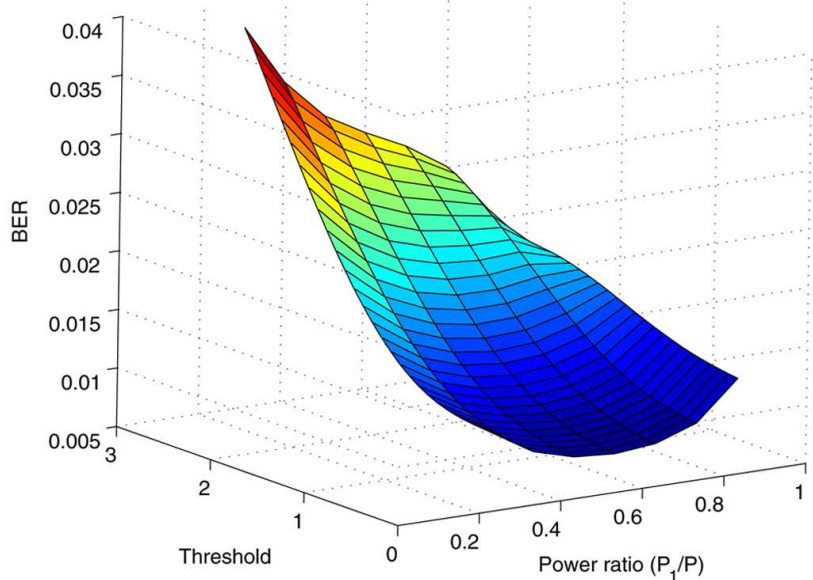

(a)

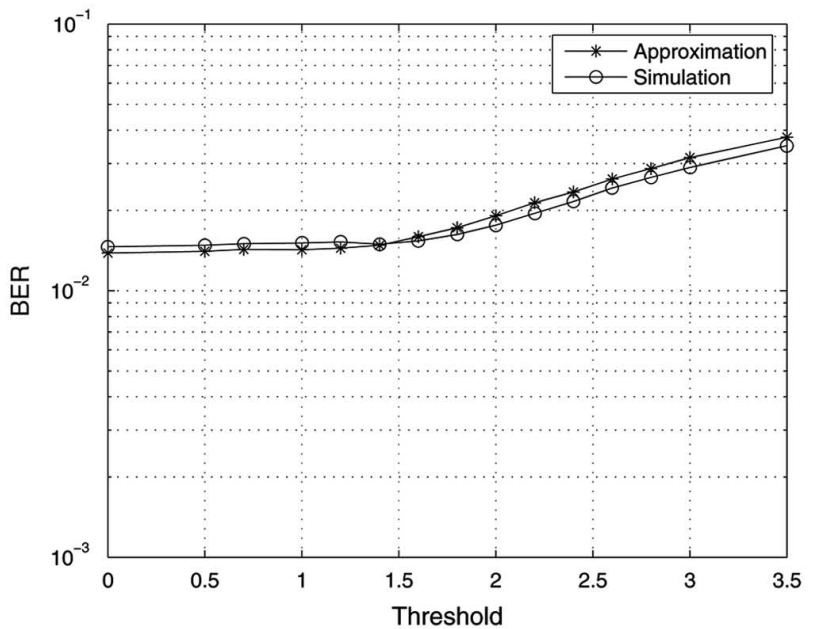

(b)

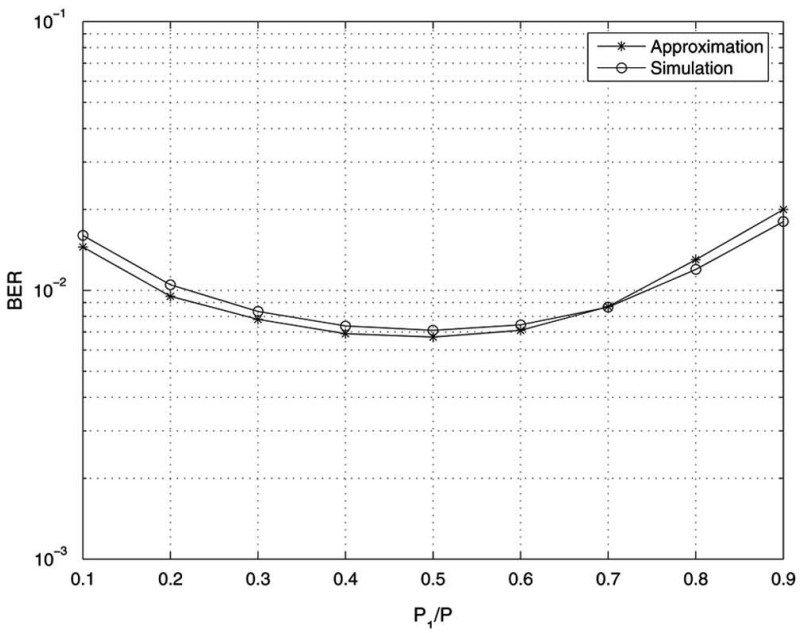

(c)

Fig. 5. DQPSK: Comparison of theoretical BER curves and simulated curves. $\sigma_{s, d}^{2}=1, \sigma_{s, r}^{2}=10$, and $\sigma_{r, d}^{2}=1$. (a) Jointly optimum threshold and power allocation. (b) Varying threshold, $P_{1}=0.5 P$ and $P_{2}=0.5 P$. (c) Varying power allocation, $\zeta=0.4$.

when the SNRs are larger than 12 and $16 \mathrm{~dB}$, respectively. This is because the proposed differential DF scheme obtains higher diversity gain but requires larger signal constellation size than

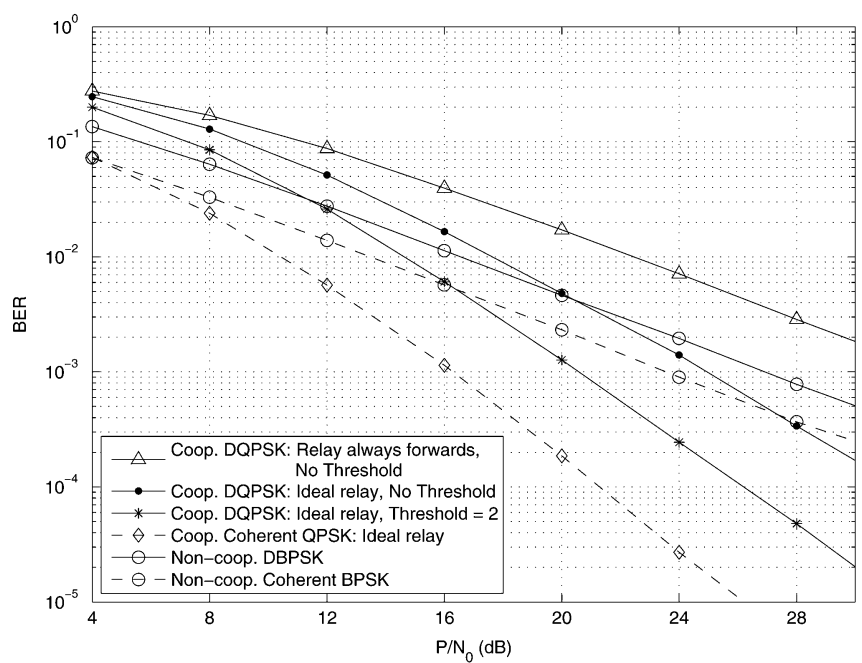

(a)

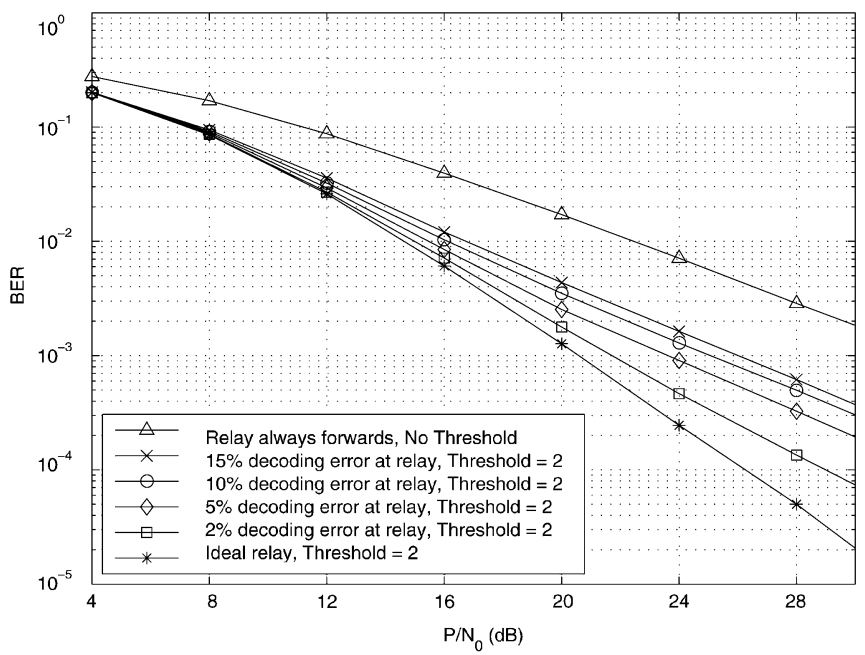

(b)

Fig. 6. DQPSK with different cooperation schemes, $P_{1}=P_{2}=$ $0.5 P, \sigma_{s, d}^{2}=1, \sigma_{s, r}^{2}=1$, and $\sigma_{r, d}^{2}=10$. (a) Comparison of different cooperation schemes. (b) Effect of decoding error at relay.

the direct transmission; the effect of noise to the system performance dominates at low SNR while the effect of diversity gain dominates at high SNR. We also show the performance of coherent DF cooperative QPSK scheme with ideal relay, which is considered as the coherent counterpart of the proposed differential DF scheme with ideal relay. Note that in the case of coherent detection, the destination is able to determine whether the relay sends an information or not from a channel estimation process, and, hence, it does not require any threshold test at the destination. At a BER of $10^{-3}$, the differential DF scheme with ideal relay but without threshold losses about $9 \mathrm{~dB}$ from the coherent counterpart, while the proposed scheme is only $5 \mathrm{~dB}$ away from the coherent counterpart.

Fig. 6(b) shows simulated performance in the case of nonideal relay, i.e., the judgment on the correctness of each decoded symbol at the relay is not perfect. The simulation scenario in Fig. 6(b) is the same as that in Fig. 6(a). In the simulation, the probability that the relay makes incorrect judgment is at $2 \%$, 
$5 \%, 10 \%$, and $15 \%$ of the total decoded symbols. It is obvious from the figure that the performance degrades as the relay makes more incorrect judgment. A small performance error floor is also observed in this case. We can see that for an incorrect judgment of $2 \%$ of the total decoded symbols, the performance curve is slightly different from the ideal relay case at medium SNR range. The effect of error propagation is quite obvious at high SNR range. Nevertheless, the obtained performance is better than the performance of the differential scheme in which the relay always forwards each decoded symbol and no threshold is used at the destination. In this case, we observe more than 8 -dB performance improvement at a BER of $10^{-3}$. The result in Fig. 6(b) indicates that it is also important to design a detection scheme at the relay that achieves small judgment errors. The problem of optimizing a threshold test at the relay and the corresponding performance analysis of the system with imperfect judgment at the relay are further investigated in our future work.

Fig. 7(a) and (b) illustrates the BER performances of the proposed scheme with different thresholds. In Fig. 7(a), the power allocation is $P_{1}=0.7 P$ and $P_{2}=0.3 P$, and the channel variances are $\sigma_{s, d}^{2}=\sigma_{s, r}^{2}=\sigma_{r, d}^{2}=1$. We can see that the proposed scheme achieves the performance diversity of two at high SNR for any threshold. However, the performance degrades as the threshold increases. Fig. 7(b) shows the BER performance in the case of power allocation $P_{1}=0.8 P$ and $P_{2}=0.2 P$, and channel variances $\sigma_{s, d}^{2}=1, \sigma_{s, r}^{2}=1$, and $\sigma_{r, d}^{2}=10$. Obviously, different thresholds result in different BER performances. The threshold of $\zeta=1.7$ provides the best performance in this scenario. Furthermore, if the threshold is too small, e.g., $\zeta=1$, not only the BER performance degrades but also the diversity order is less than two. This is because when the threshold is small, the destination tends to combine the signals from both the relay and the destination. As a result, the incorrect decoding at the relay leads to significant performance degradation at the destination.

In Fig. 8(a) and (b), we study the effect of power allocation on the BER performance for the proposed scheme with a fixed threshold. In Fig. 8(a), we consider the cooperation system with channel variances $\sigma_{s, d}^{2}=\sigma_{s, r}^{2}=\sigma_{r, d}^{2}=1$ and a threshold $\zeta=$ 2 . We can see that the power ratios $r=P_{1} / P=0.5,0.6$, and 0.7 yield almost the same performances. When the power ratio increases to $r=0.9$, the performance degradation is about $2 \mathrm{~dB}$ at BER of $10^{-4}$ compared to the equal power allocation scheme. This is due to the fact that at $r=0.9$, small power is allocated at the relay. Consequently, even though the received signal from the relay carries an information, there is high chance that its amplitude is smaller than the threshold, and, hence, the detection is based only on the received signal from the direct link. Fig. 8(b) depicts the performance in the case of channel variances $\sigma_{s, d}^{2}=$ $1, \sigma_{s, r}^{2}=1$, and $\sigma_{r, d}^{2}=10$, and a threshold $\zeta=1$. We can see that the performance improves as the power ratio increases from $r=0.5$ to $r=0.9$. The reason is that the relay-destination link is of high quality while the threshold is small. With only small power at the relay, the amplitude of the received signal from the relay can be larger than the threshold. Therefore, by allocating more power at the source, we not only increase the chance of correct decoding at the relay, but also increase the SNR of the

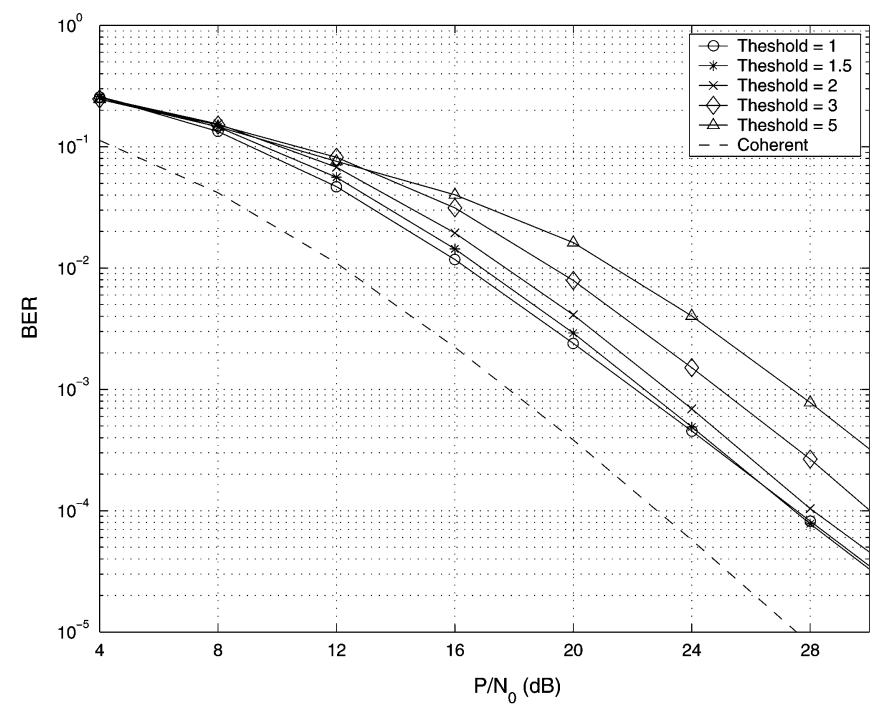

(a)

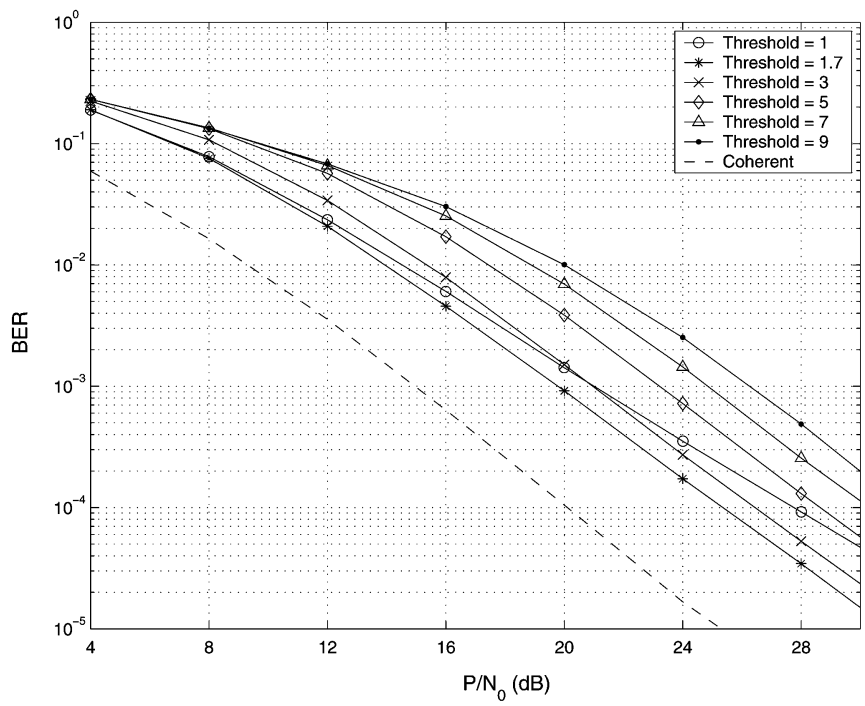

(b)

Fig. 7. DQPSK: Different thresholds with fixed power allocation. (a) $P_{1}=$ $0.7 P, P_{2}=0.3 P, \sigma_{s, d}^{2}=\sigma_{s, r}^{2}=\sigma_{r, d}^{2}=1$. (b) $P_{1}=0.8 P, P_{2}=$ $0.2 P, \sigma_{s, d}^{2}=\sigma_{s, r}^{2}=1, \sigma_{r, d}^{2}=10$.

combiner output. Based on the numerical results in Fig. 4(d), the optimum power ratio for this scenario is $r=0.9$ at the SNR of $P / \mathcal{N}_{0}=16 \mathrm{~dB}$. Clearly, the simulation results in Fig. 8(b) agree with the numerical results at the SNR of $16 \mathrm{~dB}$. Moreover, Fig. 8(b) illustrates that the power ratio of $r=0.9$ results in optimum performance for the entire SNR range. At the threshold $\zeta=1$, the proposed scheme with optimum power allocation achieves about 5-dB improvement over that with equal power allocation at a BER of $10^{-4}$.

Fig. 9(a) and (b) compares the performances of the proposed differential DF scheme with different power allocations and decision thresholds. We consider the case of $\sigma_{s, d}^{2}=\sigma_{s, r}^{2}=\sigma_{r, d}^{2}=$ 1 in Fig. 9(a), and the case of $\sigma_{s, d}^{2}=\sigma_{s, r}^{2}=1$, and $\sigma_{r, d}^{2}=10$ in Fig. 9(b). From both figures, it is clear that the proposed scheme with jointly optimum power allocation and optimum threshold yield the best performance over the entire SNR range. In the 


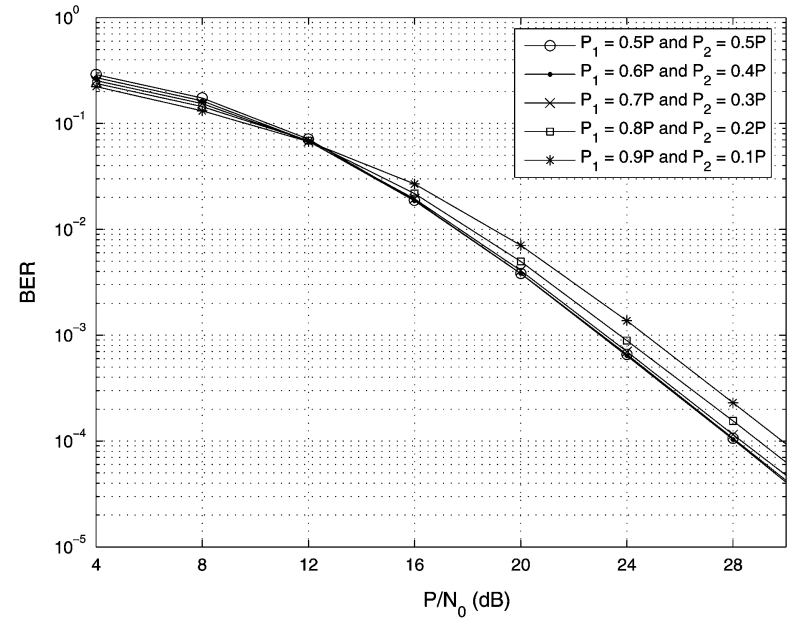

(a)

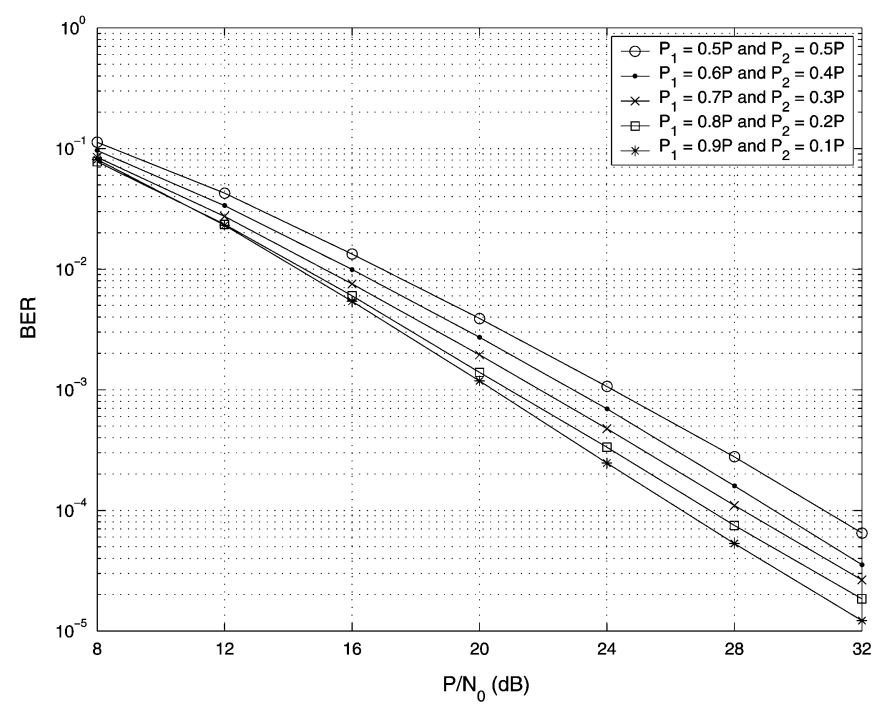

(b)

Fig. 8. DQPSK: Different power allocations with fixed threshold. (a) $\zeta=$ $2, \sigma_{s, d}^{2}=\sigma_{s, r}^{2}=\sigma_{r, d}^{2}=1$. (b) $\zeta=1, \sigma_{s, d}^{2}=\sigma_{s, r}^{2}=1, \sigma_{r, d}^{2}=10$.

case of equal link qualities, i.e., $\sigma_{s, d}^{2}=\sigma_{s, r}^{2}=\sigma_{r, d}^{2}=1$, optimum power allocation and optimum threshold yields 2-dB performance improvement at a BER of $10^{-4}$ compared to the scheme with equal power allocation and without threshold. In addition, if the power allocation is optimum, the scheme without threshold yields almost the same performance as that with optimum threshold. When the quality of the relay-destination link is very good, e.g., $\sigma_{r, d}^{2}=10$, the use of optimum threshold is more important than the use of optimum power allocation at high SNR. Specifically, by properly choosing the threshold, the proposed differential DF scheme achieves almost the same performance for any power allocation at high SNR. As we can see from Fig. 9(b), in the case of equal power allocation, using the optimum threshold leads to more than 5-dB improvement gain over the scheme without threshold at a BER of $10^{-4}$. With optimum threshold, the performance difference between the proposed scheme with optimum power allocation and that with equal power allocation is only about $0.5 \mathrm{~dB}$ at a BER of $10^{-4}$.

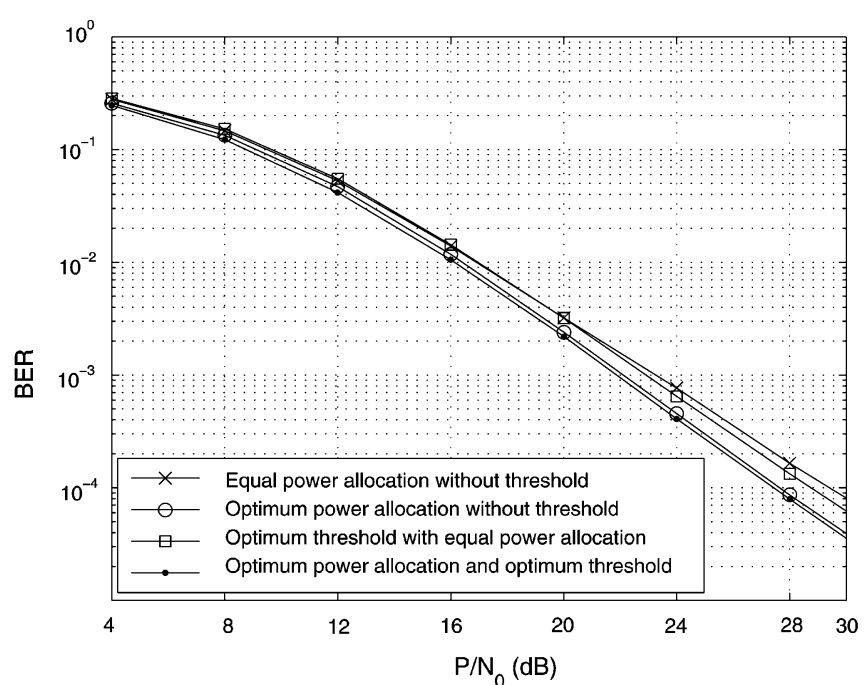

(a)

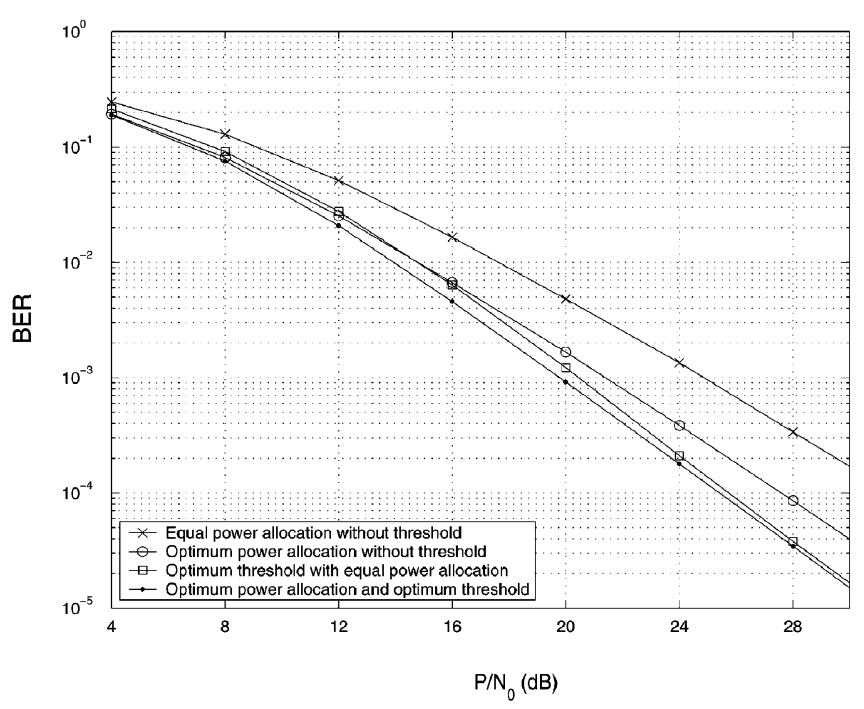

(b)

Fig. 9. DQPSK: Different power allocations and different thresholds. (a) $\sigma_{s, d}^{2}=\sigma_{s, r}^{2}=\sigma_{r, d}^{2}=1$. (b) $\sigma_{s, d}^{2}=\sigma_{s, r}^{2}=1, \sigma_{r, d}^{2}=10$.

\section{CONCLUSION}

We propose, in this paper, a threshold-based differential decode-and-forward scheme for two-user cooperative communications systems. By allowing the relay to forward only the correctly decoded symbols and introducing a decision threshold at the destination, the proposed scheme efficiently combines the signals from the direct and the relay links. We provide BER analysis of the proposed scheme with DMPSK modulation by classifying six different scenarios which lead to different instantaneous SNRs at the combiner output of the destination. The analysis focuses on the case when the relay is able to judge the correctness of each decoded symbol. A tight approximate BER expression is derived, and it serves as a performance benchmark that the proposed scheme can achieve when error propagation is negligible at the relay. The corresponding BER lower bound and BER upper bound are formulated. The approximate BER is 
very close to the simulated BER curve, and it lies between the obtained BER lower bound and BER upper bound. Based on the tight BER approximation, we determine the optimum decision threshold and power allocation numerically. Both theoretical and simulation results reveal that the optimum threshold and optimum power allocation rely on the qualities of the channel links. When the quality of the relay-destination link is much larger than the other links, i.e., $\sigma_{s, d}^{2}=\sigma_{s, r}^{2}=1$ and $\sigma_{r, d}^{2}=10$, then the decision threshold is more important than the power allocation at high SNR. For instance, in the case of DQPSK signals with equal power allocation, using the optimum threshold results in more than 5-dB improvement over the scheme with ideal relay but without threshold at a BER of $10^{-4}$. By further using the optimum power allocation, the performance improvement is about $0.5 \mathrm{~dB}$ at the same BER. Simulation results also show that the proposed scheme with DQPSK signals provides $11-\mathrm{dB}$ performance improvement over the differential DF scheme with always-forward relay and without threshold at a BER of $10^{-3}$.

\section{REFERENCES}

[1] J. G. Proakis, Digital Communications, 4th ed. New York, NY: McGraw-Hill, 2000.

[2] B. M. Hochwald and W. Sweldens, "Differential unitary space-time modulation," IEEE Trans. Commun., vol. 48, no. 12, pp. 2041-2052, Dec. 2000.

[3] B. L. Hughes, "Differential space-time modulation," IEEE Trans. Inf. Theory, vol. 46, no. 11, pp. 2567-2578, Nov. 2000.

[4] J. N. Laneman, D. N. C. Tse, and G. W. Wornell, "Cooperative diversity in wireless networks: Efficient protocols and outage behavior," IEEE Trans. Inf. Theory, vol. 50, no. 12, pp. 3062-3080, Dec. 2004.

[5] J. N. Laneman and G. W. Wornell, "Distributed space-time coded protocols for exploiting cooperative diversity in wireless networks," IEEE Trans. Inf. Theory, vol. 49, no. 10, pp. 2415-2525, Oct. 2003.

[6] J. N. Laneman, G. W. Wornell, and D. N. C. Tse, "An efficient protocol for realizing cooperative diversity in wireless networks," presented at the IEEE Int. Symp. Information Theory, Washington, DC, Jun. 2001.

[7] A. Sendonaris, E. Erkip, and B. Aazhang, "User cooperation diversity, Part I: System description," IEEE Trans. Commun., vol. 51, no. 11, pp. 1927-1938, Nov. 2003.

[8] A. Sendonaris, E. Erkip, and B. Aazhang, "User cooperation diversity, Part II: Implementation aspects and performance analysis," IEEE Trans. Commun., vol. 51, no. 11, pp. 1939-1948, Nov. 2003.

[9] M. Janani, A. Hedayat, T. E. Hunter, and A. Nosratinia, "Coded cooperation in wireless communications: Space-time transmission and iterative decoding," IEEE Trans. Signal Process., vol. 52, no. 2, pp. 362-370, Feb. 2004.

[10] W. Su, A. K. Sadek, and K. J. R. Liu, "SER performance analysis and optimum power allocation for decode-and-forward cooperation protocol in wireless networks," in Proc. IEEE WCNC, Mar. 2005, vol. 2, pp. 984-989.

[11] A. K. Sadek, W. Su, and K. J. R. Liu, "A class of cooperative communication protocols for multi-node wireless networks," in Proc. IEEE SPAWC, New York, NY, Jun. 2005, pp. 560-564.

[12] P. A. Anghel, G. Leus, and M. Kaveh, "Multi-user space-time coding in cooperative networks," in Proc. ICASSP, 2003, vol. 4, pp. 73-76.

[13] S. Barbarossa and G. Scutari, "Distributed space-time coding strategies for wideband multihop networks: Regenerative vs. nonregenerative relays," in Proc. ICASSP, 2004, vol. 4, pp. 501-504.

[14] M. O. Hasna and M.-S. Alouini, "Performance analysis of two-hop relayed transmissions over Rayleigh fading channels," in Proc. IEEE Vehicular Technology Conf., Sep. 2003, vol. 4, pp. 1992-1996.

[15] D. Chen and J. N. Laneman, "Noncoherent demodulation for cooperative diversity in wireless systems," in Proc. IEEE GLOBECOM Conf., Dallas, TX, Nov. 2004, pp. 31-35.
[16] P. Tarasak, H. Minn, and V. K. Bhargava, "Differential modulation for two-user cooperative diversity systems," presented at the IEEE GLOBECOM Conf., Dallas, TX, Nov. 2004.

[17] R. F. Pawula, "Generic error probabilities," IEEE Trans. Commun., vol. 47, no. 5, pp. 697-702, May 1999.

[18] M. K. Simon and M.-S. Alouini, "A unified approach to the probability of error for noncoherent and differentially coherent modulations over generalized fading channels," IEEE Trans. Commun., vol. 46, no. 12, pp. 1625-1638, Dec. 1998.

[19] D. G. Brennan, "Linear diversity combining techniques," Proc. IEEE, vol. 91, no. 2, pp. 331-356, Feb. 2003.

[20] H. Stark and J. W. Woods, Probability and Random Processes with Applications to Signal Processing. Upper Saddle River, NJ: PrenticeHall, 2001.

[21] G. Stuber, Principle of Mobile Communications, 2nd ed. Norwell, MA: Kluwer, 2001.

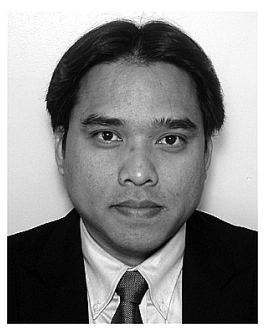

Thanongsak Himsoon (S'03-M'07) received the B.S. degree in electrical engineering from the Chulalongkorn University, Bangkok, Thailand, in 1995, and the M.S. and Ph.D. degrees in electrical engineering from the University of Maryland, College Park, in 2001 and 2006, respectively.

$\mathrm{He}$ is currently a communications systems design engineer at Meteor Communications Corporation working on algorithm and system design for wireless communications. His research interests include signal processing, wireless communications, cooperative communications, and wireless sensor networks, with particular focus on differential modulation systems. His research contributions encompass differential space-time coding and modulation for MIMO systems, MIMO-OFDM, ultrawideband systems, and cooperative communications.

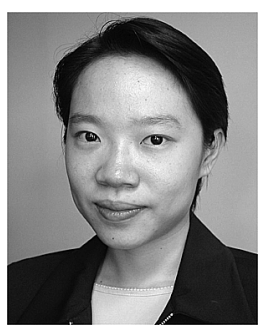

W. Pam Siriwongpairat (S'03-M'06) received the B.S. degree in electrical engineering from Chulalongkorn University, Bangkok, Thailand, in 1999, and the M.S. and Ph.D. degrees in electrical engineering from the University of Maryland, College Park, in 2001 and 2005, respectively.

She is currently a wireless communications specialist with Meteor Communications Corporation, working in the research and development of wireless communications technology. From January 2005 to May 2005, she was a Postdoctoral Research Associate with the Department of Electrical and Computer Engineering and the Institute for Systems Research (ISR), University of Maryland, College Park. Her research interests span a broad range of areas from signal processing to wireless communications and networking, including space-time coding for multi-antenna communications, cross-layer design for wireless networks, communications in mobile ad hoc networks and wireless sensor networks, OFDM systems, and ultrawideband communications.

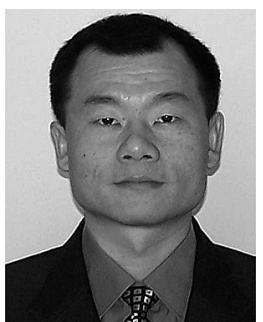

Weifeng Su (M'03) received the B.S. and Ph.D. degrees in mathematics from Nankai University, Tianjin, China, in 1994 and 1999, respectively, and the Ph.D. degree in electrical engineering from the University of Delaware, Newark, in 2002.

$\mathrm{He}$ is an Assistant Professor at the Department of Electrical Engineering, State University of New York (SUNY) at Buffalo. From June 2002 to March 2005, he was a Postdoctoral Research Associate with the Department of Electrical and Computer Engineering and the Institute for Systems Research (ISR), University of Maryland, College Park. His research interests span a broad range of areas from signal processing to wireless communications and networking, 
including space-time coding and modulation for MIMO wireless communications, MIMO-OFDM systems, cooperative communications for wireless networks, and ultrawideband (UWB) communications.

Dr. Su received the Signal Processing and Communications Faculty Award from the University of Delaware in 2002 as an outstanding graduate student in the field of signal processing and communications. In 2005, he received the Invention of the Year Award from the University of Maryland. He serves as an Associate Editor for the IEEE TRANSACTIONS ON VEHICULAR TECHNOLOGY.

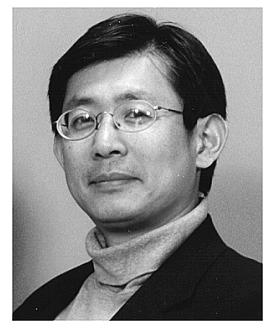

K. J. Ray Liu (F'03) is a Professor and Associate Chair of Graduate Studies and Research, Electrical and Computer Engineering Department, University of Maryland, College Park. His research contributions encompass broad aspects of wireless communications and networking, information forensics and security, multimedia communications and signal processing, bioinformatics and biomedical imaging, and signal processing algorithms and architectures.

Dr. Liu is the recipient of numerous honors and awards, including best paper awards from the IEEE Signal Processing Society (twice), the IEEE Vehicular Technology Society, and EURASIP; the IEEE Signal Processing Society Distinguished Lecturer, the EURASIP Meritorious Service Award, and National Science Foundation Young Investigator Award. $\mathrm{He}$ also received various teaching and research awards from University of Maryland, including the Poole and Kent Company Senior Faculty Teaching Award and the Invention of the Year Award. He is Vice President-Publications and on the Board of Governor of the IEEE Signal Processing Society. He was the Editor-in-Chief of IEEE Signal Processing Magazine and the founding Editor-in-Chief of the EURASIP Journal on Applied Signal Processing. 